\title{
Local Rules for Quasicrystals
}

L. S. Levitov

L. D. Landau Institute for Theoretical Physics, ul. Kosygina, dom 2, Moscow, V-334, USSR

\begin{abstract}
The relationship of local ordering and long-range order is studied for quasicrystalline tilings of plane and space. Two versions of the concept of local rules are introduced: strong and weak. Necessary conditions of the existence of strong local rules are found. They are mainly reduced to the constraints for irrational numbers related to incommensurabilities of the quasicrystals. For planar quasicrystals the quadratic irrationalities $a+b \sqrt{D}(a, b \in \mathbb{Q}, D \in \mathbb{Z})$ play an important role. For three-dimensional quasicrystals not only quadratic but also cubic irrationalities $a+b \sqrt[3]{D}+c \sqrt[3]{D^{2}}(a, b, c \in \mathbb{Q}, D \in \mathbb{Z})$ are allowed. The existence of weak local rules is established for almost all two-dimensional quasicrystals based on quadratic irrationalities and for the three-dimensional quasicrystal having icosahedral symmetry.
\end{abstract}

\section{Introduction}

Quasicrystals discovered by Schechtman, Blech, Gratias, and Cahn [1] are materials with the long-range order of a new type. They exhibit scattering properties of ideal crystals but have a point symmetry group incompatible with periodicity. Unusual diffraction properties of quasicrystals were explained with the help of periodic structures in high-dimensional spaces by Kalugin et al. [2]; Elser [3]; Duneau and Katz [4] (see also Mackay [5]; Levine and Steinhardt [6]; Kramer and Neri [7]). All the models of the atomic structure of quasicrystals discussed so far include some periodic structures in high-dimensional spaces (for the icosahedron symmetry group the dimension of the space is six and for the pentagon symmetry group it is five). The physical space is embedded into the highdimensional space as an incommensurate subspace and the positions of atoms in it are found by projection. In another version the positions of atoms are given by the common points of the periodic structure and the physical subspace. The models of this type explain successfully sharp peaks in the diffraction patterns obtained by scattering of electrons, $X$-rays and neutrons. 
However, there is some problem with all these models: they describe the longrange order without showing how it emerges from the local ordering of atoms. It is well known that the interaction of atoms has an effectively short radius, i.e. it decreases very rapidly at large distances. The short-range interaction specifies the short-range order which includes the interspacing of neighboring atoms, the angles between the segments connecting neighbors and so on. The relation of the short-range order and the long-range order in quasicrystals is not understood well enough. In this work an attempt is made to study this problem for some particular class of quasiperiodic structures (see below).

To give a heuristic introduction into the problem let us discuss first how the long-range order in an ordinary periodic crystal is supported by the short-range interaction. Consider for example the growth of a crystalline solid from the melt. When atoms get attached to the solid-liquid interface they are governed by the interaction with neighboring atoms and the place occupied by every next atom is determined by the positions of its neighbors in the solid phase. If the local arrangement of the atoms which is favoured by the growth process can be extended periodically to the whole space, then a crystal grows. It does not seem very surprising that periodic crystals can be formed by this process, since one can stabilize a periodic structure with an arbitrarily large but finite until cell by a choice of the short-range part of the interaction between atoms. To make this clear one should note that the whole structure of a crystal is completely defined by the correlation functions of atoms in the volume containing several neighboring unit cells which includes a finite number of atoms. This set of atoms gives a finite set of stability conditions, i.e. a finite set of equations for the interaction potential. These equations can undoubtedly be satisfied by a proper choice of the interaction. It is clear that the principal property of periodic structures which is responsible for the stability of the long-range order is the existence of local rules. We say that local rules exist for some structure if it is defined completely by the set of configurations of atoms having limited size, which participate in this structure.

An analogous problem for quasicrystals becomes much less trivial because the structure of quasicrystals is not periodic. Consider a quasicrystal growing from the melt. The place occupied by every atom attached to the surface of the quasicrystalline solid is again determined by some local laws based on the shortrange interatomic forces. An attached atom does not know anything about how the structure grows in the high-dimensional space. The attached atom has some information about its local environment only. Roughly speaking, one can say that atoms know only what is the set of local configurations they are allowed to form but nothing about the long-range order that will ultimately result from the growth process. In this work we do not touch upon the problem of growth of quasicrystals. The question considered here is associated with the growth problem but is somewhat easier. Rough formulation of this question is: "Is it possible to define the structure of a quasicrystal by the description of all allowed local configurations?"

For periodic crystalline structures the answer to this question is obviously affirmative. To describe unambiguously the structure of a crystal one has to give positions of the atoms occupying the unit cell and say that the structure of neighboring unit cells is the same (in other words, the unit cell is repeated periodically throughout the space). As for quasicrystals, there is no full answer to 

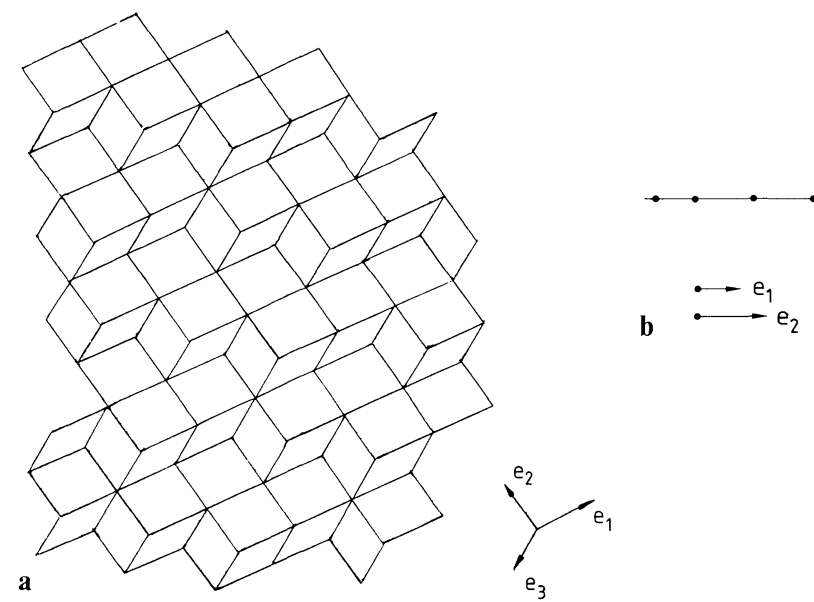

Fig. 1a and b. a Tiling of the plane associated with 3 vectors $e_{1}, e_{2}, e_{3}$. b Tiling of the line associated with 2 vectors $e_{1}, e_{2}$

the above question. All that is known concerns some special class of structures called "Penrose patterns" in honour of their inventor R. Penrose [8]. The Penrose pattern is a tiling of the plane with rhombi having pentagonal symmetry.

We consider a more general class of tilings of the plane with parallelograms defined as follows. Take $n$ nonparallel vectors $\mathbf{e}_{i}(i=1, \ldots, n)$ in the plane $\mathbb{R}^{2}$. Every pair $\left\langle\mathbf{e}_{i}, \mathbf{e}_{j}\right\rangle$ of these vectors such that $i<j$ defines the parallelogram with the sides $\mathbf{e}_{i}$ and $\mathbf{e}_{j}$. Consider the set of $n(n-1) / 2$ parallelograms generated in such a way. Suppose that the plane is divided into parallelograms so that every two adjacent parallelograms have an edge or a vertex in common (see Fig. 1a). We say that this tiling of the plane is associated with the chosen set of $n$ vectors $\mathbf{e}_{i}(i=1, \ldots, n)$ if each of the parallelograms participating in the tiling can be obtained by a shift of one of the parallelograms $\left\langle\mathbf{e}_{i}, \mathbf{e}_{j}\right\rangle(i<j)$. One can define a tiling of the 3-dimensional space $\mathbb{R}^{3}$ by a similar procedure using $n$ nonparallel vectors $\mathbf{e}_{i}(i=1, \ldots, n)$ in $\mathbb{R}^{3}$ and dividing $\mathbb{R}^{3}$ into parallelotops $\left\langle\mathbf{e}_{i}, \mathbf{e}_{j}, \mathbf{e}_{k}\right\rangle(i<j, j<k)$. The generalization to the higher dimensions is straightforward. A tiling of a line associated with a finite set of segments $\mathbf{e}_{i}(i=1, \ldots, n)$ is defined if the line is divided into segments so that each of them is identical to one of the segments $\mathbf{e}_{i}(i=1, \ldots, n)$ (see Fig. 1b). We shall denote the constituents of any tiling (parallelograms, parallelotops, segments, etc.) by the common word "tiles."

We define the lifting function $w: \mathbb{R}^{2} \rightarrow \mathbb{R}^{n}$ for an arbitrary tiling of the plane $\mathbb{R}^{2}$ associated with $n$ vectors $\mathbf{e}_{i}(i=1, \ldots, n)$. Choose an arbitrary vertex $\mathbf{x}$ of one of the parallelograms of the tiling as the origin and put $w(\mathbf{x})=(0, \ldots, 0)$. For any other vertex $\mathbf{y}$ of a parallelogram of this tiling we find a chain of the edges of the parallelograms connecting $\mathbf{x}$ and $\mathbf{y}$. Since every edge in such a chain is parallel to one of the vectors $\mathbf{e}_{i}(i=1, \ldots, n)$ we can express the vector $\mathbf{x}-\mathbf{y}$ as an integer linear combination of the vectors $\mathbf{e}_{i}$ :

$$
\mathbf{y}-\mathbf{x}=z_{1} \mathbf{e}_{1}+\ldots+z_{n} \mathbf{e}_{n}
$$

$\left(z_{i}\right.$ is equal to the number of times taken with a proper sign, when the vector $\mathbf{e}_{i}$ enters the chain.) One can immediately make sure that the integers $z_{i}(i=1, \ldots, n)$ do 
not depend on the choice of the chain connecting the vertices $\mathbf{x}$ and $\mathbf{y}$. After we put

$$
w(\mathbf{y})=\left(z_{1}, \ldots, z_{n}\right)
$$

the lifting function $w$ is defined in all vertices of the tiling. To define the function $w$ everywhere in the plane we introduce the condition of linearity (and continuity) of $w$ inside every parallelogram including its boundary. Thus obtained the lifting function $w$ transforms the tiling of $\mathbb{R}^{2}$ in a two-dimensional continuous surface in $\mathbb{R}^{n}$ consisting of two-dimensional square faces of the $n$-dimensional unit cube with the vertices being the points of the integer lattice $\mathbb{Z}^{n}$. The definition of the lifting of a two-dimensional tiling can be generalized easily for other dimensions. The image of the lifting of a $d$-dimensional tiling associated with $n$ nonparallel vectors in $\mathbb{R}^{d}$ is a $d$-dimensional continuous surface in $\mathbb{R}^{n}$, consisting of $d$-dimensional facets of the $n$-dimensional unit cubes.

Now we introduce a special class of tilings which are known as quasicrystalline tilings or, simply, quasicrystals. Consider a two-dimensional linear subspace $v$ in the $n$-dimensional space $\mathbb{R}^{n}$ given by a linear function

$$
\begin{gathered}
v: \mathbb{R}^{2} \rightarrow \mathbb{R}^{n}, \\
\left(x_{1}, x_{2}\right) \rightarrow\left(y_{1}\left(x_{1}, x_{2}\right), \ldots, y_{n}\left(x_{1}, x_{2}\right)\right), \\
y_{i}\left(x_{1}, x_{2}\right)=v_{i}\left(x_{1}, x_{2}\right)+c_{i},
\end{gathered}
$$

where $v_{i}\left(x_{1}, x_{2}\right)=a_{i} x_{1}+b_{i} x_{2} \in\left(\mathbb{R}^{2}\right)^{\prime}, i=1, \ldots, n$. We shall often denote the subspace $\operatorname{Im}[v]$ defined by (1.3) simply the letter $v$. Take an arbitrary point $\mathbf{x}=\left(x_{1}, \ldots, x_{n}\right)$ of the space $\mathbb{R}^{n}$ and attribute to it a unit cube $C[\mathbf{x}]$ with the vertices

$$
\left(x_{1} \pm \frac{1}{2}, \ldots, x_{n} \pm \frac{1}{2}\right) \text {, }
$$

where the signs in (1.4) alternate independently. The point $\mathbf{x}$ is the center of the cube $C[\mathbf{x}]$. We define the standard tube $T[v]$ associated with the subspace $v$ as the union of all the cubes $C[\mathbf{x}]$ such that $\mathbf{x}$ belongs to $v$ :

$$
T[v]=\bigcup_{\mathbf{x} \in v} C[\mathbf{x}] .
$$

Consider all the points of the lattice $\mathbb{Z}^{n}$ which belong to $T[v]$. We call the subspace $v$ "singular" if there are some integer points on the boundary of $T[v]$, otherwise $v$ is a "regular" subspace. To construct a quasicrystalline tiling of the plane we take a regular two-dimensional subspace $v$ and consider the set of integer points of $T[v]$. One can prove that there exists a tiling of the plane such that the lifting of this tiling is a two-dimensional surface included in the tube $T[v]$. Moreover, this tiling is unique if possible shifts are not taken into account (for the proof and discussion see Gahler and Rhyner [9]). We call this tiling "the quasicrystal associated with the subspace $v$," or simply " $v$-quasicrystal." One should mention that the tiling produced by this method can be either periodic or quasiperiodic depending on whether the subspace $v$ is commensurate with the lattice $\mathbb{Z}^{n}$ or not. A quasicrystal in the space of an arbitrary dimension $d$ is defined by a procedure analogous to that presented above with $v$ being a $d$-dimensional subspace of $\mathbb{R}^{n}$. Both twoand three-dimensional quasicrystalline tilings are of great interest for the physics of quasicrystals, since they are used to describe positions of atoms in real materials (see Henley and Elser [10]). 


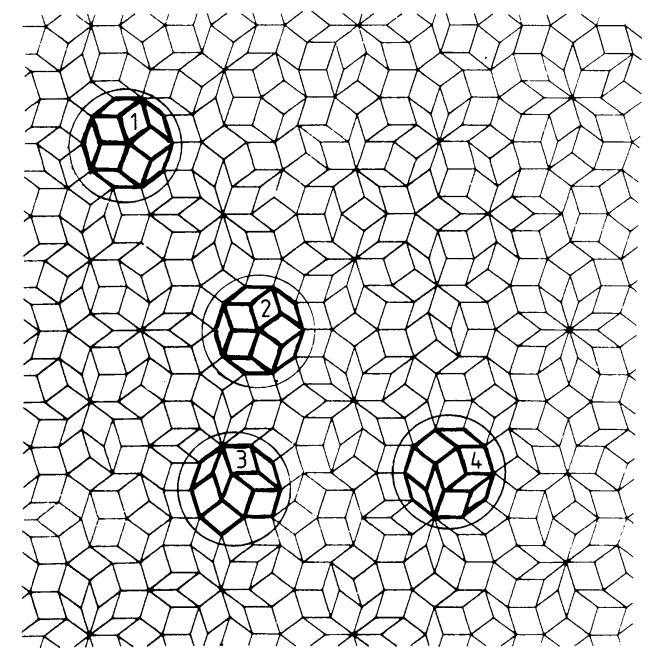

Fig. 2. Configurations of tiles 1, 2, 3, 4 inside the circles belong to the $r$-atlas ( $r$ is the radius of the circles). Configurations 1 and 2 correspond to one and the same map of the atlas

Now we introduce maps and atlases of maps for an arbitrary tiling of the plane. Define $(r, \mathbf{x})$-map as the maximal configuration of parallelograms (or tiles) lying inside the circle with the center $\mathbf{x}$ and the radius $r$. Those tiles which have common points with the boundary of the circle do not enter the map configuration (Fig. 2). Define an $r$-atlas of the tiling as the collection of all different $(s, \mathbf{x})$-maps of this tiling, such that $s<r$. If the configurations of tiles in two circles are identical they correspond to one and the same map in the atlas (Fig. 2). Since there is only a finite number of different configurations of tiles with limited size, the $r$-atlas of any tiling is a finite set of maps.

After these definitions are given we can formulate more precisely the question which is studied in this work. Suppose that after the $r$-atlas is determined for a tiling of the plane and some positive $r$, the tiling is erased and we are left with its $r$-atlas only. Is it possible to recover the original tiling in any sense? If this is possible for some finite $r$ we say that the tiling is restorable or that local rules exist for this tiling. It is clear from the consideration presented above that only the restorable tilings are of interest for physics since the non-restorable ones can hardly be stabilized by a short-range interaction. On the other hand if the tiling is restorable one can hope to find a short-range interaction of the tiles which favours this tiling.

In this work we study the problem of the existence of local rules for quasicrystalline tilings. This problem of restorability was studied by different authors for some particular examples of quasicrystals. First we mention the remarkable work by de Bruijn [11], where the existence of local rules for the Penrose tiling was proven. In the de Bruijn work the tilings of the plane with rhombi of two types (thin and thick) having angles $36^{\circ}$ and $72^{\circ}$, respectively, and supplied with arrows (see Fig. 3) were considered. It was shown that if the tiling of plane with these rhombi satisfies the "arrow condition" (the arrows on the common sides of the adjacent rhombi are identical) then it coincides exactly with 


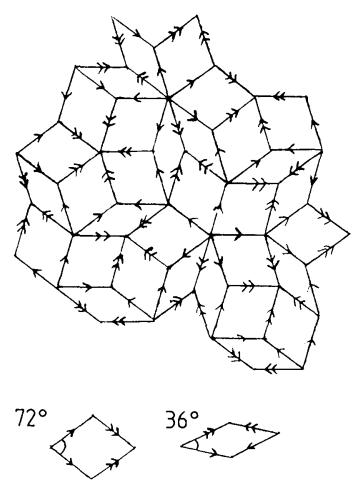

Fig. 3. Thick $\left(72^{\circ}\right)$ and thin $\left(36^{\circ}\right)$ rhombi supplied with arrows provide strong local rules for the Penrose tiling

the Penrose tiling which was originally constructed by Penrose by an entirely different algorithm (inflation-deflation procedure). In our terminology this means that local rules exist for the Penrose tiling or that it is restorable. The "arrow conditions" for the Penrose tiling were studied further by Pavlovitch and Kleman [12]. Another important contribution was made by Beenker [13], who considered the quasicrystalline tiling of the plane having eight-fold symmetry. He managed to show that there exists an arbitrarily large but finite piece of tiling for which one cannot decide using local inspection whether it participates in the quasicrystalline tiling or not. This gives strong evidence for the absence of local rules for this case.

The problem we are interested in can be formulated as: "What is the set of restorable quasicrystals?" Since the set of all atlases is countable, the set of the restorable quasicrystals is countable too. But the set of all quasicrystals is noncountable because all $d$-dimensional subspaces of an $n$-dimensional space form the Grassmann continuum and the subspaces having different slopes generate different tilings. So we conclude that most of the quasicrystals are not restorable. These arguments are strong but not constructive. A constructive description of the set of restorable quasicrystals is given below.

The contents of the paper is as follows. In Sect. 2 two different variants of the definition of the restorability property are presented: strong and weak. In accordance with these two definitions two classes of restorable quasicrystals are introduced: quasicrystals with strong local rules and quasicrystals with weak local rules. In Sect. 3 the quasicrystals having codimension one $(n=d+1)$ are considered. It is shown that they are restorable in the strong sense of Sect. 2 only if they are periodic, i.e. there are no nontrivial examples of restorable quasicrystals of codimension one. In Sect. 4 some useful properties of dual graphs of tilings are discussed. In Sect. 5 two-dimensional quasicrystals are studied. It is proven that they are restorable in the strong sense of Sect. 2 only when the coordinates $x_{1}, x_{2}$ in the plane $\mathbb{R}^{2}$ can be chosen so that all the coefficients $a_{i}, b_{i}$ $(i=1, \ldots, n)$ of the linear embedding (1.3) become quadratic irrationalities:

$$
a_{i}, b_{i} \in \mathbb{Q}(\sqrt{D})
$$


for some integer $D$ (for a more rigorous formulation see Theorem 2 and Propositions 5,6). On the other hand, it is found in Sect. 6 that almost all twodimensional quasicrystals based on quadratic irrationalities (1.6) are restorable in the weak sense of Sect. 2. Three-dimensional quasicrystals are studied in Sect. 7. The necessary condition for their restorability (in the strong sense of Sect. 2) obtained in Sect. 7 is quite analogous to the condition (1.6) for the two-dimensional tilings. The main difference is that not only quadratic irrationalities, but also cubic irrationalities of the fields $\mathbb{Q}(\sqrt[3]{D})$ are allowed. The quasicrystalline tiling of the three-dimensional space having icosahedral symmetry is studied at the end of Sect.7. This tiling is shown to be restorable in the weak sense of Sect. 2. The preliminary version of this work containing no rigorous proofs of the assertions but only formulations of basic definitions and statements was published in [14].

Notation. The letters $\mathbb{R}, \mathbb{Z}, \mathbb{Q}$ have the usual meaning of real line, set of integers and set of rational numbers.

If $x \in \mathbb{R}$ then $[x]$ is the integer part of $x$.

$\mathbb{R}^{n}$ is the linear space of $\left(x_{1}, \ldots, x_{n}\right)$, where $x_{i} \in \mathbb{R}(i=1, \ldots, n)$, with the standard scalar product and metrics:

$$
(\mathbf{x}, \mathbf{y})=x_{1} y_{1}+\ldots+x_{n} y_{n}, \quad\|\mathbf{x}\|^{2}=x_{1}^{2}+\ldots+x_{n}^{2},
$$

where $\mathbf{x}=\left(x_{1}, \ldots, x_{n}\right), \mathbf{y}=\left(y_{1}, \ldots, y_{n}\right) \in \mathbb{R}^{n}$.

$\mathbb{Z}^{n}$ is the integer lattice $\left(z_{1}, \ldots, z_{n}\right)$, where $z_{i} \in \mathbb{Z}(i=1, \ldots, n)$.

The letter $i$ always represents an element of the set $\{1,2, \ldots, n\}$. "For all $i$ " will mean for $i=1, \ldots, n$.

$\mathbb{R}^{n \prime}=\left(\mathbb{R}^{n}\right)^{\prime}$ denotes the space of linear functions

$$
f: \mathbb{R}^{n} \rightarrow \mathbb{R}, \quad\left(x_{1}, \ldots, x_{n}\right) \rightarrow f_{1} x_{1}+\ldots+f_{n} x_{n},
$$

where $f_{i} \in \mathbb{R}$ and $x_{i}$ are the standard coordinates in $\mathbb{R}^{n}$. $\mathbb{Q}^{n \prime}=\left(\mathbb{Q}^{n}\right)^{\prime}$ is the space of linear functions (1.8) with rational coefficients $f_{i} \in \mathbb{Q}$. Elements of $\mathbb{Q}^{n \prime}$ form a linear space over the field $\mathbb{Q}$.

For the dimension of a linear space $v$ the standard notation $\operatorname{dim}(v)$ is used.

For every integer $D$ we use the notation $\mathbb{Q}(\sqrt{D})$ for the field of all $a+b \sqrt{D}$ $(a, b \in \mathbb{Q})$ and the notation $\mathbb{Q}(\sqrt[3]{D})$ for the field of all $a+b \sqrt[3]{D}+c \sqrt[3]{D^{2}}(a, b, c \in \mathbb{Q})$.

For two functions $f, g$ we use " $f * g$ " to denote their composition.

\section{Basic Definitions}

Consider $n$ nonparallel vectors $\mathbf{e}_{i}$ in the plane $\mathbb{R}^{2}$ and closed parallelograms (or tiles) associated with the pairs of vectors $\left\langle\mathbf{e}_{i}, \mathbf{e}_{j}\right\rangle(i<j)$. We define a configuration of tiles as a finite set of parailelograms in the plane satisfying the tiling conditions:

a) every tile of the configuration is produced by a proper shift of one of the parallelograms $\left\langle\mathbf{e}_{i}, \mathbf{e}_{j}\right\rangle(i<j)$;

b) if two tiles of the configuration intersect, then the intersection set is either a vertex or an edge;

c) the configuration is a connected set.

Some examples of configurations are shown in Fig. 4. The radius of $a$ configuration is defined as the minimal radius of the circle containing this 

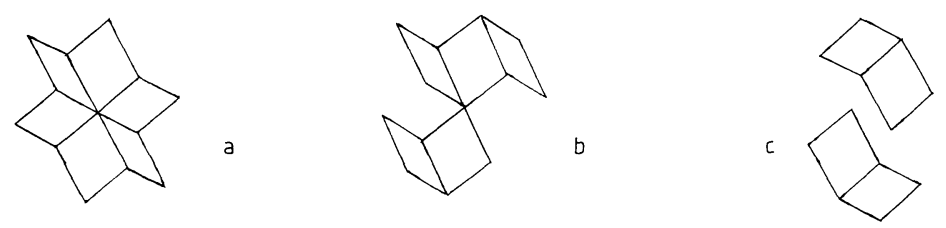

Fig. $\mathbf{4 a - c}$. Examples of configurations to tiles: $\mathbf{a}$ and $\mathbf{b}$ are configurations, while $\mathbf{c}$ is not

configuration. We say that two configurations are identical if there exists a shift transforming one configuration onto another.

We define r-rules as an arbitrary set of nonidentical configurations having radius less than $r$. It is clear that there exist only a finite number of different $r$-rules for every fixed $r$.

When a positive $r$ is given and some $r$-rules are specified we say that a tiling of the plane satisfies these $r$-rules if for every positive $s, s<r$, the $s$-atlas of this tiling is a subset of the $r$-rules.

Now we introduce local rules (LR) for planar quasicrystalline tilings. Let some $r$-rules, $r>0$, be specified. We call them strong local rules if they have the following properties.

a) every tiling satisfying these $r$-rules is a quasicrystal;

b) at least one quasicrystal satisfies the rules;

c) Let two subspaces $v$ and $v^{\prime}$ define quasicrystals according to (1.3), (1.4), (1.5). If both $v$-quasicrystal and $v^{\prime}$-quasicrystal satisfy the $r$-rules then the subspaces $v$ and $v^{\prime}$ are parallel (i.e. they are identical up to a shift).

Remark. If the embedding functions $v, v^{\prime}$ define parallel subspaces according to (1.3) (the parameters being $a_{i}, b_{i}, c_{i}$, and $a_{i}^{\prime}, b_{i}^{\prime}, c_{i}^{\prime}$ respectively) then one can find a coordinate transformation $x_{1}^{\prime}=\alpha x_{1}+\beta x_{2}, x_{2}^{\prime}=\gamma x_{1}+\delta x_{2}$ such that $a^{\prime}=\alpha a+\beta b$, $b^{\prime}=\gamma a+\delta b$.

In other words, strong LR fix the slope of the embedding (1.3). When strong LR exist they specify the structure almost entirely with the only freedom left related to shifts of the subspace (i.e. to the changes of constants $c_{i}$ in (1.3)]. The importance of such shifts becomes clear from the following proposition.

Proposition 1. For every linear embedding $v: \mathbb{R}^{2} \rightarrow \mathbb{R}^{n}$ given by (1.3) consider a linear subspace $\mathbb{Q}^{n \prime}[v] \subset\left(\mathbb{Q}^{n}\right)^{\prime}$ consisting of all functions $f \in\left(\mathbb{Q}^{n}\right)^{\prime}$ such that the composition $f * v$ is a constant function on $\mathbb{R}^{2}: f(v(\mathbf{x}))=$ const for all $\mathbf{x} \in \mathbb{R}^{2}$. Let two embeddings $v^{\prime}$ and $v^{\prime \prime}$ be defined by (1.3) with the coefficients $a_{i}^{\prime}, b_{i}^{\prime}, c_{i}^{\prime}$ and $a_{i}^{\prime \prime}, b_{i}^{\prime \prime}, c_{i}^{\prime \prime}$ respectively.

If $a_{i}^{\prime}=a_{i}^{\prime \prime}, b_{i}^{\prime}=b_{i}^{\prime \prime}$ for all $i$, and $f\left(\mathbf{c}^{\prime}\right)=f\left(\mathbf{c}^{\prime \prime}\right)$ for all linear functions $f \in \mathbb{Q}^{n \prime}[v]$, where $\mathbf{c}^{\prime}=\left(c_{1}^{\prime}, \ldots, c_{n}^{\prime}\right), \mathbf{c}^{\prime \prime}=\left(c_{1}^{\prime \prime}, \ldots, c_{n}^{\prime \prime}\right)$, then for every positive $r$ the $r$-atlas of the $v^{\prime}$ quasicrystal coincides with the r-atlas of the $V^{\prime \prime}$-quasicrystal.

We shall prove this proposition in Sect. 4 after dual graphs of tilings will be introduced. Some comments should be made on the definition of the subspace $\mathbb{Q}^{n \prime}[v]$. The dimension of $\mathbb{Q}^{n \prime}[v]$ is equal to the "number of commensurabilities" of the linear embedding $v$. If $a_{i} \in \mathbb{Q}$ and $b_{i} \in \mathbb{Q}$ in (1.3) for all $i$, then $\operatorname{dim}\left(\mathbb{Q}^{n \prime}[v]\right)=n-2$. If $v$ is completely incommensurate then $\operatorname{dim}\left(\mathbb{Q}^{n \prime}[v]\right)=0$. To finish with strong LR we formulate without proof a simple assertion on periodic tilings (a planar tiling is periodic if it has two noncollinear periods). 
Proposition 2. For any periodic tiling one can find $r>0$ and $r$-rules such that

a) this tiling satisfies the rules;

b) every two tilings satisfying the rules are related by a shift of the plane $\mathbb{R}^{2}$.

This proposition means simply that a periodic tiling is completely described by its $r$-atlas for sufficiently large $r$. As a consequence, we obtain that periodic quasicrystals satisfy strong LR.

In addition to strong LR we introduce weak LR. If $v$ is a linear imbedding given by (1.3) we say that the $v$-quasicrystal satisfies weak local rules if there exist positive $r$ and $r$-rules such that

a) the $v$-quasicrystal satisfies these $r$-rules;

b) for any tiling satisfying the $r$-rules and every lifting of this tiling there exists a positive constant $C$ such that the distance from any point of the lifting to the subspace $\operatorname{Im}[v]$ is not larger than $C$ (the distance is measured in the standard metric of $\mathbb{R}^{n}$ ).

In other words, the lifting of every tiling satisfying weak LR is almost parallel to the subspace $\operatorname{Im}[v]$ and the distance between the points of the lifting and corresponding points of the subspace $\operatorname{Im}[v]$ is uniformly bounded by a constant. In the physical language weak LR allow some structural disorder without destroying the long-range quasicrystalline order.

The extension of all the constructions and definitions introduced above to arbitrary dimensions is straightforward.

In order to have an example of using these definitions we consider onedimensional quasicrystals which are generated by linear embeddings of $\mathbb{R}$ in $\mathbb{R}^{n}$ (by analogy with (1.3)]:

$$
v: \mathbb{R} \rightarrow \mathbb{R}^{n}, \quad x \rightarrow\left(a_{1} x+c_{1}, \ldots, a_{n} x+c_{n}\right) ; \quad c \in \mathbb{R} .
$$

A fairly simple assertion is valid:

Proposition 3. A one-dimensional quasicrystal (2.1) satisfies weak LR only if it is periodic: $a_{i} / a_{j} \in \mathbb{Q}$ for all $i$ and $j$ in (2.1).

Proof. Suppose that some weak LR for the quasicrystal (2.1) do exist. Let $r$ denote the maximal radius of the configurations included in the rules.

Suppose that a tiling of the line $\mathbb{R}$ satisfying these LR is given. Find two nonintersecting pieces in this tiling with the configurations of tiles in the pieces being identical and having radius larger than $r$. Let $g$ be the shift of the line $\mathbb{R}$ $(x \rightarrow x+g)$ which transforms one of the pieces onto another.

Consider a new periodic tiling with the period equal to $g$ which is generated by a repetition of the region between the two pieces of the original tiling. One checks immediately that so obtained periodic tiling satisfies the LR. By the definition of weak LR the lifting of the tiling we started with does not go far from the lifting of the constructed periodic tiling. Since the lifting of the periodic tiling goes along a commensurate subspace, the linear embedding (2.1) for the original tiling is commensurate. Hence the original tiling is periodic. Proposition 3 is proven.

In conclusion I would like to comment on the definition of local rules. The existence of strong LR implies the existence of weak LR and the absence of weak LR implies the absence of strong LR. 


\section{Quasicrystals of Codimension One}

In this section we study quasicrystalline tilings of $\mathbb{R}^{n-1}$ generated by linear embeddings of $\mathbb{R}^{n-1}$ in $\mathbb{R}^{n}$. The most interesting cases for physics are $n=3,4$. The $n=3$-case corresponds to the plane in the three-dimensional space, i.e. describes a surface of a periodic crystal (see [15]). Every linear embedding $\mathbb{R}^{n-1} \rightarrow \mathbb{R}^{n}$ gives a hyperplane in $\mathbb{R}^{n}$ and, on the other hand, after the vectors $\mathbf{e}_{i}$ in $\mathbb{R}^{n-1}$ are specified (that we always have in mind) every hyperplane defines a quasicrystalline tiling of $\mathbb{R}^{n-1}$.

Now we characterize the hyperplanes which yield periodic tilings of $\mathbb{R}^{n-1}$. Consider a linear embedding

$$
\begin{gathered}
v: \mathbb{R}^{n-1} \rightarrow \mathbb{R}^{n}, \\
\mathbf{x} \rightarrow\left(f_{1}(\mathbf{x})+c_{1}, \ldots, f_{n}(\mathbf{x})+c\right) ; \quad \mathbf{x} \in \mathbb{R}^{n-1}, f \in\left(\mathbb{R}^{n-1}\right)^{\prime} .
\end{gathered}
$$

Any hyperplane in $\mathbb{R}^{n}$ parallel to the hyperplane $\operatorname{Im}[v]$ is given by an equation

$$
g(\mathbf{y})=\text { const }\left(\mathbf{y} \in \mathbb{R}^{n}, g \in\left(\mathbb{R}^{n}\right)^{\prime}\right),
$$

where $g$ is connected with $v$ by

$$
g * v=\text { const } .
$$

Consider the set $\mathbb{Z}[g]$ of vectors of $\mathbb{Z}^{n}$ which are parallel to the hyperplane $g$ :

$$
\mathbb{Z}[g]=\left\{\mathbf{y} \mid \mathbf{y} \in \mathbb{Z}^{n}, g(\mathbf{y})=0\right\} .
$$

$\mathbb{Z}[g]$ is a sublattice of $\mathbb{Z}^{n}$. We say that the hyperplane $g$ is rational if $\operatorname{dim}(\mathbb{Z}[g])=n-1$, i.e. if this hyperplane is spanned by $n-1$ independent vectors of $\mathbb{Z}^{n}$. When $\operatorname{Im}[v]$ is a rational hyperplane the corresponding $v$-quasicrystal is periodic. One should mention the duality of the subspace $\mathbb{Q}^{n \prime}[v]$ defined in Proposition 1 and the lattice $\mathbb{Z}[v]$. Thus introduced objects $g$ and $\mathbb{Z}[g]$ will be useful for the proof of the principal statement of this section formulated as

Theorem 1. If an embedding $v: \mathbb{R}^{n-1} \rightarrow \mathbb{R}^{n}$ generates a quasicrystal satisfying some strong LR, then the quasicrystal periodic.

This statement means that nonperiodic quasicrystals of codimension one cannot satisfy strong LR. Let us explain briefly the idea of the proof. We take an incommensurate embedding $v$ and for an arbitrarily large positive $r$ find a new embedding $u$ such that the slopes of the subspaces $\operatorname{Im}[u]$ and $\operatorname{Im}[v]$ are different but close to each other and the $r$-atlases of the two quasicrystals are identical. Since the obtained subspaces with different slopes are not parallel, an incommensurate quasicrystal with strong LR cannot exist.

In the proof of Theorem 1 we use the following notations. If a hyperplane $g$ is an image of an embedding $v$ we write " $g$-quasicrystal" instead of " $v$-quasicrystal," since we assume the set of vectors $\mathbf{e}_{i}$ in $\mathbb{R}^{n-1}$ to be specified (see above). We also write " $(r, v)$-atlas" instead of " $r$-atlas of the $v$ quasicrystal."

Proof of Theorem 1. Consider two regular (see Sect. 1) parallel hyperplanes $g^{\prime}, g^{\prime \prime}$ in $\mathbb{R}^{n}$ and find $r$-atlases of the corresponding quasicrystals for some $r>0$. According to Proposition 1 we obtain that these $r$-atlases coincide. This means that the $r$-atlas depends on the linear part of the embedding (1.3) only and does not 
depend on the constants $c_{i}$. This remark shows that an atlas is well defined on the Grassmann space $G[n, n-1]$ of hyperplanes in $\mathbb{R}^{n}$.

Definition. Consider an arbitrary configuration $k$ of tiles in $\mathbb{R}^{n-1}$ and attribute a subset $\Gamma[k] \in G[n, n-1]$ to it. $\Gamma[k]$ is defined as the set of all hyperplanes $g$ such that $g$-quasicrystal contains the configuration $k$ infinitely many times.

Note that whatever the configuration $k$ is the set $\Gamma[k]$ is open in the topology of $G[n, n-1]$ since no integer points can lie on the boundary of a standard tube (see Sect. 1).

Consider an arbitrary configuration $k$ of tiles and a hyperplane $g$. There are three possibilities for $g$ :

a) the point $g$ of $G[n, n-1]$ lies inside the open set $\Gamma[k]$;

b) the point $g$ of $G[n, n-1]$ lies on the boundary of the set $\Gamma[k]$;

c) the point $g$ of $G[n, n-1]$ belongs to the exterior of the set $\Gamma[k]$.

In the case a) of configuration $k$ belongs to the $(r, g)$-atlas for $r$ large enough. In the case b) we say that "the configuration $k$ is dangerous for the hyperplane $g$."

Let us give a geometrical description of dangerous configurations. Consider a hyperplane $g$ and write (3.2) in the standard coordinates $\left(y_{1}, \ldots, y_{n}\right)$ of $\mathbb{R}^{n}$ :

$$
g_{1} y_{1}+\ldots+g_{n} y_{n}=c .
$$

The points of the tube $T[g]$ are given by

$$
c-g^{\prime}<g_{1} y_{1}+\ldots+g_{n} y_{n}<c+g^{\prime}, \quad \text { where } g^{\prime}=\left(\left|g_{1}\right|+\ldots+\left|g_{n}\right|\right) / 2 .
$$

The boundary of $T[g]$ consists of two hyperplanes

$$
\begin{aligned}
& g_{1} y_{1}+\ldots+g_{n} y_{n}=c+g^{\prime}, \\
& g_{1} y_{1}+\ldots+g_{n} y_{n}=c-g^{\prime} .
\end{aligned}
$$

b)

Let a configuration $k$ be dangerous for the hyperplane $g$. Consider its lifting $w(k)$. From the definition of a dangerous configuration we obtain that $w(k)$ can be immersed in the closure of the tube (3.6) by a proper shift. After this shifting is performed the lifting $w(k)$ is positioned so that both boundaries (3.7a) and (3.7b) contain corner points of $w(k)$ (see Fig. 5). Consider the set $A$ of the corner points of $w(k)$ which belong to the hyperplane (3.7a) and the set $B$ of the corner points

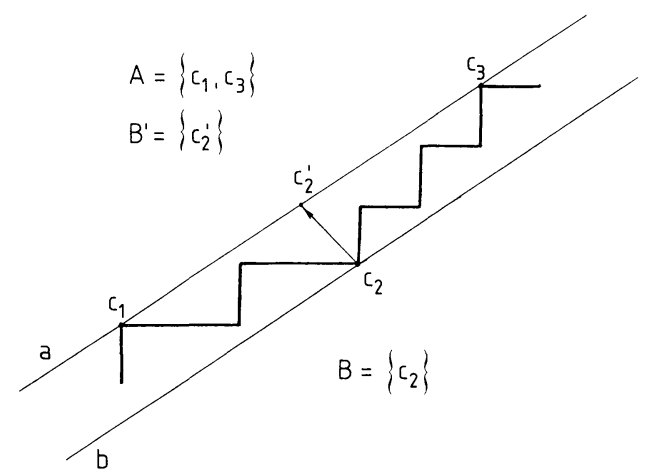

Fig. 5. Dangerous configuration immersed in the tube is shown [the boundary hyperplanes (3.7) are represented by the lines $a$ and $b]$. Dangerous corner points $C_{1}, C_{2}, C_{3}$ form two sets $A, B$. Vector $\overrightarrow{C_{2} C_{2}^{\prime}}$ represents the shift (3.8) 
belonging to the hyperplane (3.7b). Since the shift

$$
\mathbf{y} \rightarrow \mathbf{y}+\left(\operatorname{sign}\left(g_{1}\right), \ldots, \operatorname{sign}\left(g_{n}\right)\right) \quad\left(\mathbf{y} \in \mathbb{R}^{n}\right)
$$

transforms the hyperplane (3.7b) onto the hyperplane (3.7a), the set $B^{\prime}$ produced by the shift (3.8) of the set $B$ belongs to the hyperplane (3.7a) (Fig. 5). Consider the set $S$ of all vectors connecting the points of the union $A \cup B^{\prime}$. Since the set $A$ contains at least one point and the set $B$ also contains at least one point, the set $S$ is not empty. Two properties of the set $S$ are important for us:

a) all the vectors belonging to $S$ are parallel to the hyperplane $g$;

b) each vector of $S$ belongs to $\mathbb{Z}^{n}$.

The property a) is true because the hyperplane (3.7a) is parallel to $g$. The property b) is true because every vector connecting corner points of a configuration belongs to $\mathbb{Z}^{n}$ and the shift vector in (3.8) also belongs to $\mathbb{Z}^{n}$. Consider the sublattice $H[k]$ of $\mathbb{Z}^{n}$ formed by all integer linear combinations of the vectors belonging to $S$. The sublattice $H[k]$ is parallel to the hyperplane $g$ and has the dimension not less than one. Thus we conclude that every configuration $k$ dangerous for a hyperplane yields a nonzero sublattice $H[k] \subset \mathbb{Z}^{n}$ parallel to this hyperplane.

To obtain the proof of Theorem 1 we first show that if a configuration $k$ is of the type a) or c) for a hyperplane $g$ (see above), then $k$ is of the type a) or c) for every hyperplane close to $g$ in the topology of $G[n, n-1]$. Consider a configuration $k$ of the type a). The point $g$ of the space $G[n, n-1]$ of hyperplanes lies inside the set $\Gamma[k]$ since this set is open. This means that every hyperplane $u$ close enough to $g$ lies inside $\Gamma[k]$ or, in other words, that the configuration $k$ is of the type a) for the hyperplane $u$. Since the exterior of a set is an open set, the same arguments are applicable for the configurations of the type c).

Let us take a nonrational hyperplane $g(\operatorname{dim}(\mathbb{Z}[g])<n-1)$. Choose some positive $r$ and consider all configurations $\left\{k_{j}\right\}(j=1, \ldots, N)$ dangerous for $g$ and having radii less than $r$. Let $H[r]$ be the sum of all the sublattices $H\left[k_{j}\right] . H[r]$ is the minimal sublattice of $\mathbb{Z}^{n}$ which includes all $H\left[k_{j}\right](j=1, \ldots, N)$. From the other hand $H[r]$ is included in the maximal sublattice $\mathbb{Z}[g]$ defined above. It is clear that $H[r]=\mathbb{Z}[g]$ if $r$ is large enough. Take a hyperplane $u$ such that

(i) $\mathbb{Z}[u]=\mathbb{Z}[g]$,

(ii) $u$ is close but not equal to $g$.

This is possible only if the hyperplane $g$ is nonrational, i.e. if $\operatorname{dim}(\mathbb{Z}[g])<n-1$. If $u$ is close enough to $g$, then all the configurations $k_{j}$ dangerous for $g$ are dangerous for $u$ also.

We have shown that for a nonrational hyperplane $g$ and sufficiently large $r$ a hyperplane $u$ exists such that every configuration with the radius less than $r$, which is of the type a), b), or c) for $g$, is of the same type for $u$. This means that the $(r, g)$-atlas and the $(r, u)$-atlas are identical. Theorem 1 is proven.

Our final remark is that the method of this proof is not applicable for the case of codimension larger than one. The key point of the proof is that the boundary of the tube $T[g]$ consists of two parallel hyperplanes (3.7) connected by an integer vector. This makes it possible to gather all the critical corner points of a dangerous configuration on one of the two boundary hyperplanes. If the codimension is larger than one, this method fails since different parts of the tube boundary are not parallel. 


\section{Dual Graphs and Grids}

In this section we introduce dual graphs of tilings and study their properties with the purpose of application in Sects. 5-7.

We consider the tilings of $\mathbb{R}^{2}$ as graphs and introduce their dual graphs by the standard definition used in the graph theory [16] (see Fig. 6). The dual graph of a tiling consists of $n$ arrays of curves in one-to-one correspondence with the vectors $\mathbf{e}_{i}$. The important properties of the dual graph are:

a) no two curves of one array intersect;

b) every two curves of different arrays intersect and have one point in common;

c) no three curves have a common point;

d) the intersection points of pairs of curves are in one-to-one correspondence with the tiles and, moreover, the intersections of the curves belonging to the $i^{\text {th }}$ and $j^{\text {th }}$ arrays correspond to the parallelograms $\left\langle\mathbf{e}_{i}, \mathbf{e}_{j}\right\rangle$ for all $i, j=1, \ldots, n$.

The dual graph of a tiling defines it completely up to the shift, after the correspondence of the vectors $\mathbf{e}_{i}$ and the arrays of lines of the graph is specified (that we always assume). Dual graphs yielding identical tilings are referred to as equivalent.

Remark. Any continuous one-to-one transformation $\mathbb{R}^{2} \rightarrow \mathbb{R}^{2}$ does not destroy the topology of the graph and, hence, transforms it to an equivalent one.

Now we discuss the properties of dual graphs of quasicrystalline tilings first studied by de Bruijn. In the work [11] de Bruijn shows that the dual graph of the Penrose tiling is equivalent to that consisting of five arrays of straight equidistant lines with the angles between the lines of different arrays being multiples of $36^{\circ}$. It was shown later that an analogous assertion is valid for an arbitrary quasicrystal [9]. One should note that for a dual graph of an arbitrary tiling of $\mathbb{R}^{2}$ it is possible to find a continuous transformation $\mathbb{R}^{2} \rightarrow \mathbb{R}^{2}$ such that it transforms two of the arrays of the curves of the graph onto two arrays of straight parallel equidistant lines. The characteristic property of dual graphs of quasicrystals is that a continuous transformation can be found such that not only two but all the arrays of curves are simultaneously transformed onto arrays of straight parallel equidistant lines (Fig. 6.b).

An array of straight parallel and equidistant lines is called "grid." Every grid is given by a linear function $f \in \mathbb{R}^{2 \prime}$ and equations

$$
f(\mathbf{x})=k+c, \quad\left(\mathbf{x} \in \mathbb{R}^{2}\right),
$$

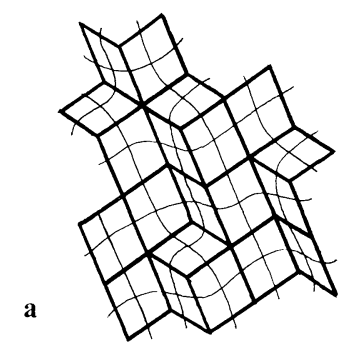

Fig. 6a and b. a A piece of a quasicrystalline tiling and its dual graph; $\mathbf{b}$ The lines of the dual graph a transformed into grids by a continuous transformation 
where $k$ takes all integer values and $c$ is some real constant. The dual graph of a quasicrystal is given by a set of functions $\left\{f_{i}\right\}$, a set of constants $\left\{c_{i}\right\}$ and equations

$$
f_{i}(\mathbf{x})=k_{i}+c_{i}, \quad\left(\mathbf{x} \in \mathbb{R}^{2}, k_{i} \in \mathbb{Z}\right) .
$$

Remark. Consider $v$-quasicrystal corresponding to the embedding (1.3). Let its dual graph be given by (4.2). One can prove that a linear transformation $\mathbb{R}^{2} \rightarrow \mathbb{R}^{2}$ exists such that it transforms $v_{i}(\mathbf{x})$ in $f_{i}(\mathbf{x})$ for all $i$ (see [9]).

Another important remark is that it is possible to reformulate all the constructions introduced in Sects. 1,2 in terms of dual graphs. One can take a graph with the properties a), b), c) (see above) as the fundamental object instead of tilings and define liftings, maps, atlases, strong LR and weak LR for graphs. We briefly show below how to do this.

Let us begin with the lifting of a dual graph. The lifting of a graph having properties a), b), c) is defined as a function

$$
w: \mathbb{R}^{2} \rightarrow \mathbb{R}^{2}, \quad \mathbf{y} \rightarrow\left(w_{1}(\mathbf{y}), \ldots, w_{n}(\mathbf{y})\right), \quad\left(\mathbf{y} \in \mathbb{R}^{2}\right),
$$

where the component functions $w$ are defined as follows. Remove all the curves of the graph except those from the $i^{\text {th }}$ array. Choose a point $\mathbf{x}$ which does not belong to the graph and set $w_{i}(\mathbf{x})=0$. For any other point $\mathbf{y}$ of the plane, the value $w_{i}(\mathbf{y})$ is given by the number of curves from the $i^{\text {th }}$ array separating the points $\mathbf{x}$ and $\mathbf{y}$, taken with a proper sign (Fig. 7). The function $w_{i}$ is defined everywhere except the lines of the $i^{\text {th }}$ array. Hence, the lifting $w(\mathbf{y})$ is defined for all points $\mathbf{y}$ of $\mathbb{R}^{2}$ not belonging to the graph.

The relation of the lifting of a graph to the lifting of the corresponding tiling is quite clear. Every region surrounded by the lines of the graph corresponds to some vertex of the tiling. Since all the components $w_{i}$ of (4.3) take constant integer values in such a region, the function with integer values is defined on the vertices of the tiling. After adding the condition of linearity inside the tiles (see Sect. 1) one gets the lifting of the tiling. On the other hand, the lifting of a tiling defines unambiguously the lifting of its dual graph. We denote both liftings by the letter $w$ since it will be clear everywhere below which of the two is used. Finally we give the expression for the lifting $w$ of the dual graph (4.2) of a quasicrystal:

$$
w_{i}(\mathbf{y})=\left[f_{i}(\mathbf{y})-c_{i}\right] \quad\left(\mathbf{y} \in \mathbb{R}^{2}\right) .
$$

Maps of a dual graph are defined as the maps of the corresponding tiling. After maps are defined so the definitions of $r$-atlases, rules, strong RL, and weak RL are straightforward.

Fig. 7.

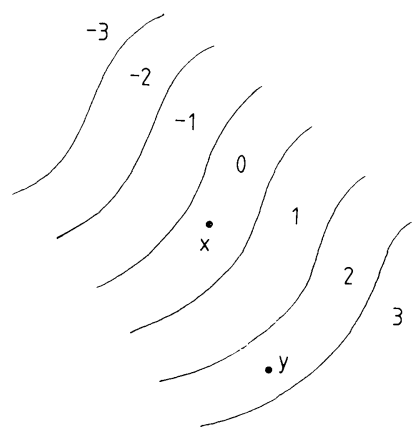


Now we apply the introduced concepts to prove Proposition 1.

Proof of Proposition 1. For the proof we make use of a theorem proven by Kronecker (see [20]).

Kronecker Theorem. Let $k$ linear functions $L_{j} \in\left(\mathbb{R}^{m}\right)^{\prime}(j=1, \ldots, k)$ and an arbitrary vector $\mathbf{g} \in \mathbb{R}^{k}\left(\mathbf{g}=\left(g_{1}, \ldots, g_{k}\right)\right)$ be given. The following two statements are equivalent.

(i) For every $\varepsilon>0$ one can find an integer vector $\mathbf{a} \in \mathbb{Z}^{m}$ such that the inequalities

$$
\left\langle\left\langle L_{j}(\mathbf{a})-g_{j}\right\rangle\right\rangle<\varepsilon
$$

are satisfied simultaneously for all $j=1, \ldots, k$. (Here $\langle\langle x\rangle$ means $\min (\{x\},\{-x\})$, i.e. the distance from $x$ to the nearest integer; $\{x\}=x-[x]$ is the fractional part of $x$.)

(ii) For every integer vector $\mathbf{z} \in \mathbb{Z}^{k}\left(\mathbf{z}=\left(z_{1}, \ldots, z_{k}\right)\right)$ such that the linear function

$$
z_{1} L_{1}(\mathbf{x})+\ldots+z_{k} L_{k}(\mathbf{x})
$$

has integer coefficients, the quantity

takes an integer value.

$$
z_{1} g_{1}+\ldots+z_{k} g_{k}
$$

In order to apply this theorem we first find two independent functions among $v_{i}$ in (1.3). Let them be $v_{1}$ and $v_{2}$. Since the equations

$$
t_{1}=v_{1}\left(x_{1}, x_{2}\right), \quad t_{2}=v_{1}\left(x_{1}, x_{2}\right)
$$

can be solved for all $t_{1}, t_{2}$ two independent linear functions

are defined. We denote

$$
x_{1}\left(t_{1}, t_{2}\right), \quad x_{2}\left(t_{1}, t_{2}\right)
$$

$$
\mathbf{c}=\mathbf{c}^{\prime}-\mathbf{c}^{\prime \prime}=\left(c_{1}^{\prime}-c_{1}^{\prime \prime}, \ldots, c_{n}^{\prime}-c_{n}^{\prime \prime}\right)=\left(c_{1}, \ldots, c_{n}\right),
$$

and choose the functions $\xi, \eta$ given by

$$
\begin{aligned}
& \xi=t_{1}+c_{1}=v_{1}\left(x_{1}, x_{2}\right)+c_{1}, \\
& \eta=t_{2}+c_{2}=v_{2}\left(x_{1}, x_{2}\right)+c_{2},
\end{aligned}
$$

as new coordinates in $\mathbb{R}^{2}$. Consider all $y_{i}$ [see (1.3)] as functions of these new variables:

$$
y_{1}(\xi, \eta)=\xi, \quad y_{2}(\xi, \eta)=\eta,
$$

for $i>2$ :

$$
y_{i}(\xi, \eta)=v_{i}\left(x_{1}(\xi, \eta), x_{2}(\xi, \eta)\right)+v_{i}\left(x_{1}^{0}, x_{2}^{0}\right)+c_{i},
$$

where $x_{1}^{0}, x_{2}^{0}$ satisfy the equations

$$
v_{1}\left(x_{1}^{0}, x_{2}^{0}\right)=-c_{1}, \quad v_{2}\left(x_{1}^{0}, x_{2}^{0}\right)=-c_{2} .
$$

It is easy to see that the functions

$$
L_{j}(\xi, \eta)=v_{j+2}\left(x_{1}(\xi, \eta), x_{2}(\xi, \eta)\right) \quad(j=1, \ldots, n-2),
$$

and the vector

$$
\mathbf{g}=\left(v_{3}\left(x_{1}^{0}, x_{2}^{0}\right)+c_{3}, \ldots, v_{n}\left(x_{1}^{0}, x_{2}^{0}\right)+c_{n}\right) \in \mathbb{R}^{n-2}
$$


satisfy Condition (ii) of the Kronecker theorem $(m=2, k=n-2)$. The theorem states that for every $\varepsilon>0$, one can find $\xi, \eta \in \mathbb{Z}$ such that

and hence

$$
\left\langle\left\langle v_{j}\left(x_{1}(\xi, \eta), x_{2}(\xi, \eta)\right)-v_{j}\left(x_{1}^{0}, x_{2}^{0}\right)-c_{j}\right\rangle\right)<\varepsilon,
$$

$$
\left.\left\langle v_{j}\left(x_{1}(\xi, \eta)-x_{1}^{0}, x_{2}(\xi, \eta)-x_{2}^{0}\right)-c_{j}\right\rangle\right\rangle<\varepsilon
$$

for all $j=3, \ldots, n$.

We see that for every pair of vectors $c^{\prime}, c^{\prime \prime}$ with the properties claimed in Proposition 1 and an arbitrary point $\mathbf{x}^{\prime}=\left(x_{1}^{\prime}, x_{2}^{\prime}\right) \in \mathbb{R}^{2}$, one can find another point $\mathbf{x}^{\prime \prime}=\left(x_{1}^{\prime \prime}, x_{2}^{\prime \prime}\right) \in \mathbb{R}^{2}$ such that

$$
\left\langle\left\langle v_{i}\left(\mathbf{x}^{\prime}\right)+c_{i}^{\prime}-v_{i}\left(\mathbf{x}^{\prime \prime}\right)-c_{i}^{\prime \prime}\right\rangle\right\rangle \varepsilon
$$

for all $i=1, \ldots, n$ [according to (4.11) $\left.x_{1}^{\prime \prime}=x_{1}^{\prime}+x_{1}^{0}-x_{1}(\xi, \eta), x_{2}^{\prime \prime}=x_{2}^{\prime}+x_{2}^{0}-x_{2}(\xi, \eta)\right]$.

After the inequalities (4.12) are viewed as conditions for the lifting functions and combined with the properties of the dual graphs discussed above, we obtain that one can find $r>0$ depending on $\varepsilon$ such that

a) $\left(r, x^{\prime}\right)$-map of the $v^{\prime}$-quasicrystal coincides with $\left(r, x^{\prime \prime}\right)$-map of the $v^{\prime \prime}$-quasicrystal.

b) $r \rightarrow \infty$, when $\varepsilon \rightarrow 0$.

Properties a) and b) together mean that the $r$-atlas of the $v^{\prime}$-quasicrystal coincides with the $r$-atlas of the $v^{\prime \prime}$-quasicrystal. Proposition 1 is proven.

A useful assertion concerning the relation of distances between the intersection points of a dual graph and the corresponding parallelograms of the tiling should be finally mentioned.

Proposition 4. Consider a quasicrystalline tiling and its dual graph formed by grids. One can find four constants $c, c^{\prime}, C, C^{\prime} \in \mathbb{R}\left(C>0, C^{\prime}>0\right)$ such that for any two intersection points $\mathbf{x}, \mathbf{y} \in \mathbb{R}^{2}$ of the lines of the graph and corresponding parallelograms of the tiling with centers $\mathbf{x}^{\prime}, \mathbf{y}^{\prime} \in \mathbb{R}^{2}$, the following inequalities are satisfied:

$$
C\|\mathbf{y}-\mathbf{x}\|+c<\left\|\mathbf{y}^{\prime}-\mathbf{x}^{\prime}\right\|<C^{\prime}\|\mathbf{y}-\mathbf{x}\|+c^{\prime},
$$

where $\|\ldots\|$ is the standard metrics in $\mathbb{R}^{2}$.

This proposition (which we leave without proof) means that the metrics in the "dual graph representation" of a quasicrystal is equivalent to the metrics in the "tiling representation." Proposition 4 will be used in Sect. 6.

\section{Two-Dimensional Quasicrystals}

In this section we prove the absence of strong LR for a wide class of quasicrystalline tilings of the plane. First introduce an important definition. Consider three linear functions $f, g, h \in\left(\mathbb{R}^{2}\right)^{\prime}$.

Definition. We say that the SI-condition is satisfied for the triplet $(f, g, h)$ if there exist $k, l, m \in \mathbb{Z}$ such that $|k|+|l|+|m|>0$ and the system of equations

$$
f(\mathbf{x})=k, \quad g(\mathbf{x})=l, \quad h(\mathbf{x})=m, \quad\left(\mathbf{x} \in \mathbb{R}^{2}\right)
$$

has a solution (the abbreviation SI = "second intersection" will become clear later). 
It is useful to write the SI-condition (5.1) explicitly in the coordinate representation. If the functions $f, g, h$ have the form

$$
\begin{aligned}
& f(\mathbf{x})=f_{1} x_{1}+f_{2} x_{2}, \quad g(\mathbf{x})=g_{1} x_{1}+g_{2} x_{2}, \\
& h(\mathbf{x})=h_{1} x_{1}+h_{2} x_{2},
\end{aligned}
$$

the SI-condition means that there exist integers $k, l, m$ such that $|k|+|l|+|m|>0$ and

$$
\operatorname{det}\left\|\begin{array}{lll}
f_{1} & f_{2} & k \\
g_{1} & g_{2} & l \\
h_{1} & h_{2} & m
\end{array}\right\|=0 .
$$

After the functions $f, g, h$ are chosen the SI-condition (5.3) is no more than a linear equation for $k, l, m \in \mathbb{Z}$. The set of the integer solutions of Eq. (5.3) is a sublattice of the integer lattice $\mathbb{Z}^{3}$. The dimension $d$ of this sublattice is an important characteristic of the triplet $(f, g, h)$. If $d=3$, then $f, g$ and $h$ are collinear in $\mathbb{R}^{2 \prime}$ :

$$
f(\mathbf{x})=\operatorname{const} g(\mathbf{x}), \quad g(\mathbf{x})=\text { const } h(\mathbf{x})
$$

for all $\mathbf{x} \in \mathbb{R}^{2}$. If $d=2$, then the functions $f, g, h$ are linearly dependent over the field $\mathbb{Q}$, i.e. there exist rational numbers $a, b, c \in \mathbb{Q}$ such that $|a|+|b|+|c|>0$ and

$$
a f(\mathbf{x})=b g(\mathbf{x})+\operatorname{ch}(\mathbf{x})=0
$$

for all $\mathbf{x} \in \mathbb{R}^{2}$. If $d=0$ then the SI-condition is not satisfied. The most interesting $d=1$-case has a simple geometric meaning. Consider three grids

$$
f(\mathbf{x})=c_{1}+k, \quad g(\mathbf{x})=c_{2}+l, \quad h(\mathbf{x})=c_{3}+m,
$$

where $k, l, m$ take integer values and $c_{1}, c_{2}, c_{3}$ are some constants. Suppose that the SI-condition is satisfied for the triplet $f, g, h$ and the constants $c_{1}, c_{2}, c_{3}$ in (5.6) are chosen so that three of the grid lines have an intersection point. The condition (5.1) means that there is a second intersection point common for other three lines of these grids. If $d=1$, these triple intersection points form a row (Fig. 8). A pattern of three grids satisfying the SI-condition has at least one period.

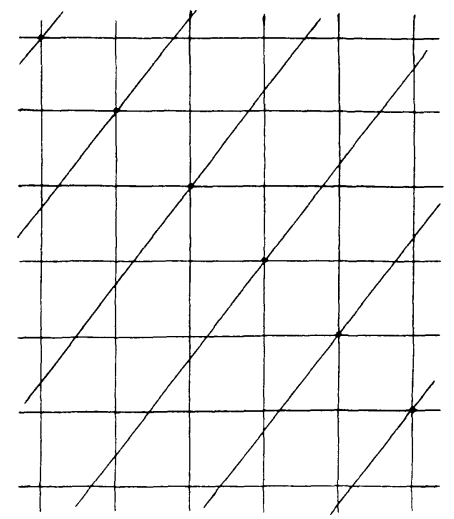

Fig. 8. When three grids satisfying SI-condition intersect, the intersection points form a row 
The principal assertion of this section is:

Theorem 2. Let a quasicrystalline tiling of the plane be generated by grids (4.2). If this quasicrystal has strong LR, then the SI-condition is satisfied for every triplet of the grids (4.2).

We begin the proof of Theorem 2 with several auxiliary lemmas.

Lemma 1. Suppose the plane is decomposed onto two half-planes. If two quasicrystals are identical in one of the half-planes, they are identical everywhere.

The assertion of Lemma 1 is a trivial consequence of the quasiperiodicity of quasicrystalline tilings.

Lemma 2. Let a two-dimensional quasicrystal satisfy some strong LR. Let $r$ be the maximal radius of the configurations included in these LR. If two positive numbers $s, q \in \mathbb{R}$ and two points $\mathbf{x}, \mathbf{y}$ of the plane $\mathbb{R}^{2}$ exist for which

a) the restrictions of the tiling to two rings

are identical;

$$
\begin{aligned}
& s<\|\mathbf{z}-\mathbf{x}\|<s+2 q, \\
& s<\|\mathbf{z}-\mathbf{y}\|<s+2 q, \quad\left(\mathbf{z} \in \mathbb{R}^{2}\right)
\end{aligned}
$$

b) the $(s, \mathbf{x})$-map is different from the $(s, \mathbf{y})$-map, then the radius $r$ is bigger than $q: r>q$.

Proof of Lemma 2. Suppose the maximal radius $r$ of the strong LR is not larger than $q: r<q$. Make a new tiling by the replacement of the $(s+2 q, \mathbf{x})$-map by the $(s+2 q, \mathbf{y})$-map. Since the new tiling satisfies the same LR, it is a quasicrystal. Thus obtained two quasicrystals contradict Lemma 1. This proves Lemma 2.

Remark. One can easily reformulate Lemma 2 for grids, since there is a simple relation for distances between points of the dual graph and distances between corresponding parallelograms of the tiling (see Proposition 4).

We also need some results on the triangles formed by grids. Consider three grids (5.6) and the triangles formed by the lines of these grids. All such triangles are similar. We say that a triangle has diameter $d$ if the largest of the distances between the vertices of the triangle is equal to $d$.

Choose a small positive $d$ and consider the triangles having diameter less than $d$. We are interested in the distribution of such triangles over the plane.

Suppose first that the functions $f, g, h$ are linearly dependent over $\mathbb{Q}$ [see (5.5)]. In this case there exists a positive $d$ such that the diameter of any triangle is larger than $d$. So the triangles with sufficiently small diameters are absent.

If the functions $f, g, h$ are linearly independent over $\mathbb{Q}$, the triangles with small diameters do exist. Their distribution is characterized by the following lemmas.

Lemma 3. Define the distribution density $n(d)$ of the triangles as

$$
n(d)=\lim _{S \rightarrow \infty} N / S,
$$

where $N$ is the number of triangles having diameters less than $d$ and lying in a large circle of the area $S$. Thus defined the function $n(d)$ is linear, i.e. $n=$ const $d$. 

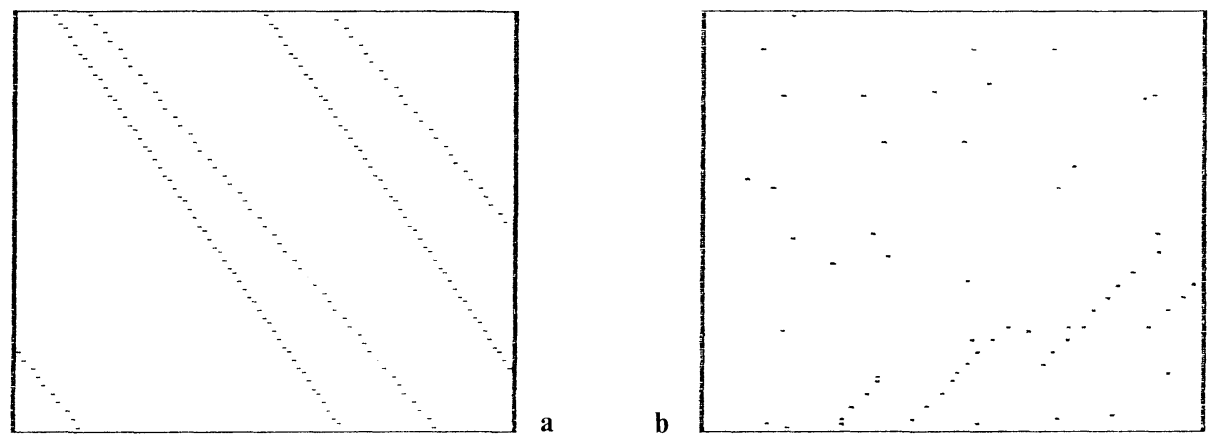

Fig. 9a and b. Distribution of small triangles formed by three grids over the plane. a SI-condition is satisfied; b SI-condition is not satisfied

Lemma 4. If the SI-condition is satisfied for $f, g, h$ and the number $d$ is small enough, then the triangles having diameter less thand form parallel rows (Fig. 9a) such that

a) the distance between every two neighboring triangles in a row is a constant not depending on $d$ and the row;

b) the minimal separation of every two neighboring rows, as a function of $d$, tends to infinity when $d$ goes to zero.

Lemma 5. Define the function $\operatorname{dist}(d)$ as the minimal distance between the triangles having diameters less than $d$. If the SI-condition is not satisfied for the functions $f, g, h$, then

$$
\operatorname{dim}_{d \rightarrow 0} \operatorname{dist}(d)=\infty \text {. }
$$

The assertion of Lemma 5 means that small triangles are far from each other, if the SI-condition is not satisfied (Fig. 9b).

One easily obtains the proof of Lemmas 3, 4, 5 by making use of the expression for the area $S$ of a triangle formed by three lines $a_{j} x_{1}+b_{j} x_{2}=c_{j}(j=1,2,3)$ :

$$
\begin{gathered}
S=\frac{\left|\Delta_{123}\right|^{2}}{2 \Delta_{12} \Delta_{23} \Delta_{31}}, \quad \text { where } \Delta_{123}=\operatorname{det}\left\|\begin{array}{lll}
a_{1} & b_{1} & c_{1} \\
a_{2} & b_{2} & c_{2} \\
a_{3} & b_{3} & c_{3}
\end{array}\right\|, \\
\Delta_{j k}=\operatorname{det}\left\|\begin{array}{ll}
a_{j} & a_{k} \\
b_{j} & b_{k}
\end{array}\right\|, \quad j, k=1,2,3 .
\end{gathered}
$$

Consider a $v$-quasicrystal for some embedding $v$ and its dual graph given by the grids (4.2). The subspace $\mathbb{Q}^{n \prime}[v]$ defined in Proposition 1 characterizes the shifts of the grids which do not change the atlases of the tiling.

Definition. We define an equivalence transformation for a quasicrystal (4.2) as a transformation

$$
c_{i}^{\prime}=c_{i}+h_{i}
$$

of the parameters $c_{i}$ in (4.2) such that

$$
f(h)=0 \text { for all } f \in \mathbb{Q}^{n^{\prime}}[v] ; \quad h=\left(h_{1}, \ldots, h_{n}\right) .
$$


a)

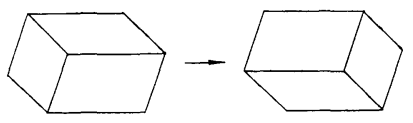

b)
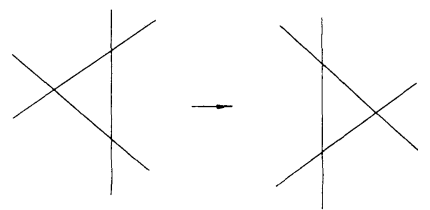

Fig. 10a and $\mathbf{b}$. Rearrangements produced in the tiling $\mathbf{a}$ and in the grid pattern $\mathbf{b}$ by an equivalence transformation (5.10), (5.11)

The transformation (5.10), (5.11) changes the tiling but preserves its $r$-atlases for all $r$ (see Proposition 1).

We are interested now in what the grids pattern changes are that are produced by the equivalence transformations (5.10), (5.11). Since there are

$$
N=C_{n}^{3}=n(n-1)(n-2) / 6
$$

subsets of cardinality three in a set of cardinality $n$, the triplets of the grids (4.2) form triangles of $N$ types. When an equivalence transformation is performed, some of the triangles are rearranged (Fig. 10). It is clear that equivalence transformations (5.10) with small $h_{i}$ yield rearrangements of small triangles only. Several properties of the rearranging triangles will be useful for the proof of Theorem 2. By making use of (5.9) one proves easily the following assertions.

Lemma 6. Let the absolute values of all $h_{i}$ in (5.10) be smaller than some positive d:

$$
\left|h_{i}\right|<d \text {. }
$$

$A$ constant $C$ exists such that all the triangles rearranged by the equivalence transformation (5.10), (5.11) have diameters less than $C d$.

Lemma 7. Let three grid functions $f_{i}, f_{j}, f_{k}(i, j, k=1, \ldots, n)$ from (4.2) be linearly dependent over $\mathbb{Q}$. There is no equivalence transformation that yields rearrangements of the triangles formed by the lines of the $i^{\text {th }}$, the $j^{\text {th }}$ and the $k^{\text {th }}$ grids. The only result of an equivalence transformation is a shift of such triangles without any change of their size.

Lemma 8. Let three grid functions $f_{i}, f_{j}, f_{k}(i, j, k=1, \ldots, n)$ from (4.2) be linearly independent over $\mathbb{Q}$. There exists a positive constant $C$ such that for every sufficiently small positive d one can choose the parameters $h_{i}$ of the transformation (5.10), (5.11) so that

a) $\left|h_{i}\right|<C d$ for all $i$;

b) every triangle with the diameter less than $d$ formed by the lines of the $i^{\text {th }}$, the $j^{\text {th }}$, and the $k^{\text {th }}$ grids is rearranged by one of the two equivalence transformations

$$
c_{i}^{\prime}=c_{i}+h_{i}, \quad c_{i}^{\prime}=c_{i}-h_{i} .
$$

Everything is ready now for the proof of Theorem 2 . The general plan of this proof is the following. For a quasicrystal having at least one triplet of grids not satisfying the SI-condition we find an equivalence transformation (5.10), (5.11) with sufficiently small $h_{i}$ such that

a) there exists a triangle which is rearranged by this transformation;

b) the piece of the tiling in a thick ring surrounding this triangle is not rearranged. 
Since equivalence transformations do not change the atlases of tilings, the rearranged piece of the tiling inside the ring can be found in the original tiling. After this we apply Lemma 2 which states that if strong LR exist, then there is a configuration included in these rules with the radius larger than half of the thickness of the ring. Since one can make the thickness of the ring arbitrarily large by a proper choice of small $h$, we conclude that strong LR do not exist.

Proof of Theorem 2. Let $r$ be the maximal radius of the configurations from the LR of a quasicrystal. Divide the triplets of grids (4.2) forming the dual graph of the quasicrystal into three sets: $A, B$, and $C$. The set $A$ contains the triplets which do not satisfy the SI-condition. The set $C$ contains the triplets of functions which are linearly dependent over $\mathbb{Q}$. The set $B$ contains the triplets which satisfy the SIcondition but are not included in the set $C$. We call a triangle formed by a triplet of grids belonging to the set $A(B, C)$ " $A$-triangle" (" $B$-triangle," " $C$-triangle"). For every triplet of grids of the set $A$ the function dist $: \mathbb{R} \rightarrow \mathbb{R}$ is defined according to Lemma 5. We define the function Dist: $\mathbb{R} \rightarrow \mathbb{R}$ by

$$
\operatorname{Dist}(d)=\min \operatorname{dist}(d) \text {. }
$$

The minimum in (5.16) is taken over all triplets belonging to $A$.

Suppose that Theorem 2 is not valid, i.e. the set $A$ is not empty but contains at least one triplet, say $f_{1}, f_{2}, f_{3}$. Let $d$ be some small positive number (we will choose the exact value of $d$ at the end of the proof). Choose the parameters $h_{i}$ in the equivalence transformation (5.10), (5.11) according to Lemma 8. Consider the triangles which are rearranged by the transformations (5.15).

The triangles which are rearranged by any of two transformations (5.15) have diameters less than const $d$ (Lemma 6). No $C$-triangles are rearranged (Lemma 7). The $B$-triangles which are rearranged by the transformations (5.15) form rows separated by large distances and, hence, rarely distributed over the plane (Lemma 4).

For every rearranged $A$-triangle we find its separation from the nearest rearranged $B$-triangle. The maximum of these distances, taken over all rearranged $A$-triangles, defines the function $\operatorname{Dist}^{\prime}(d)$. Let us prove that

$$
\lim _{d \rightarrow 0} \operatorname{Dist}^{\prime}(d)=\infty \text {. }
$$

Suppose that (5.17) is not valid, i.e. a constant $D$ exists such that $\operatorname{Dist}^{\prime}(d)<D$ for all $d$. This means that each of the rearranged $A$-triangles is separated by a distance less than $D$ from one of the rearranged $B$-triangles. Consider the union of all $D$-vicinities of the rearranged $B$-triangles ( $D$-vicinity of a triangle is the set of all points separated from it by less than $D$ ). One can cover this set with strips of the width $2 D+d$ centered on the rows (Fig. 11). Since the minimal distance between parallel rows of $B$-triangles becomes arbitrarily large when $d$ gets sufficiently small (Lemma 4), the parallel strips do not intersect for $d$ small enough. Since the distance between every two similar rearranged $A$-triangles is not less than

$$
\operatorname{Dist}(\text { const } d \text { ) }
$$

[see (5.16)], one can estimate the density $n$ of these $A$-triangles as

$$
n<\text { const }^{\prime}(2 D+d) / \text { Dist }(\text { const } d)
$$


Fig. 11

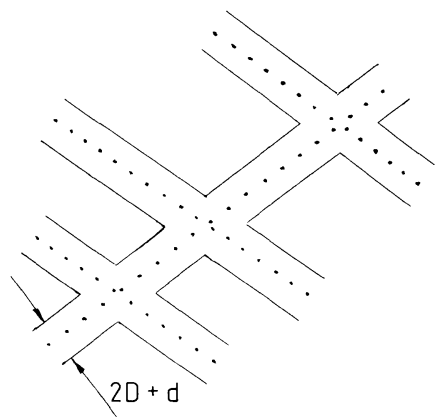

for sufficiently small $d$. The constant in (5.18) is equal to the product of the constants from Lemma 6 and Lemma 8. Since

$$
\lim _{x \rightarrow 0} \operatorname{Dist}(x)=\infty
$$

(see Lemma 9), the inequality (5.19) is in conflict with Lemma 3. This contradiction proves the assertion (5.17).

Find a rearranged $A$-triangle such that all the rearranged $B$-triangles are separated from this triangle by the distances larger than $\operatorname{Dist}^{\prime}(d) / 2$. Let

$$
X=\min \left[\operatorname{Dist}(\operatorname{const} d), \operatorname{Dist}^{\prime}(d) / 2\right],
$$

where the constant in (5.21) is equal to that in (5.18). Choose a circle having the radius $X / 2$ with the center $\mathbf{y}$ inside the found $A$-triangle. No rearranged $B$-triangles are contained in this circle. There is not more than one rearranged $A$-triangle for each triplet of the set $A$ inside the circle. Therefore, there are not more than $N$ rearranged triangles in this circle $[N$ is given by $(5.13)]$. Divide the circle into $2 N+1$ concentric rings

$$
\frac{j X}{(2 N+1) 2} \leqq|\mathbf{x}-\mathbf{y}|<\frac{(j+1) X}{(2 N+1) 2},
$$

where $\mathbf{x} \in \mathbb{R}^{2}, j=0, \ldots, 2 N$. If $d$ is small enough, every rearranged triangle inside the circle intersects not more than two rings (5.22). Since the number of these triangles is not more than $N$, at least one of the rings (5.22) is free of rearranged triangles.

Consider the piece of the grid pattern inside the thus found free ring. This piece is rearranged by one of the equivalence transformations (5.15). Since the equivalence transformations do not change atlases, the rearranged piece can be found somewhere else in the original grid pattern. One can apply Lemma 2 and conclude that the radius $r$ is not less than the half of the thickness of the ring, i.e. that

$$
r>\text { const } \frac{X}{4(2 N+1)} \text {. }
$$

A constant appears in (5.23) because the quantity $r$ mentioned in Lemma 2 is the radius of configurations of tiles but not of pieces of the grid pattern (see Proposition 4).

Since the quantity $X$ can be made unlimitedly large by a proper choice of $d$ [see (5.17) and (5.20)], we obtain the contradiction with finiteness of $r$. 
Theorem 2 is proven.

Let us apply Theorem 2 to the quasicrystals having $n$-fold rotational symmetry. $n$ vectors $\mathbf{e}_{i}$ in this case form a regular star and the grid functions $f_{i}$ from (4.2) are given by

$$
f_{i}\left(x_{1}, x_{2}\right)=\cos (\alpha j) x_{1}+\sin (\alpha j) x_{2}, \quad \alpha=2 \pi / n,
$$

where $j=0, \ldots, n-1$. Let us take the triplet $f_{0}, f_{1}, f_{3}$ and check whether it satisfies the SI-condition or not. The SI-condition yields

$$
\operatorname{det}\left\|\begin{array}{ccc}
1 & 0 & n_{1} \\
\cos \alpha & \sin \alpha & n_{2} \\
\cos 3 \alpha & \sin 3 \alpha & n_{3}
\end{array}\right\|=0,
$$

where $n_{1}, n_{2}, n_{3}$ are some integers, or

$$
n_{1} \sin 2 \alpha-n_{2} \sin 3 \alpha+n_{3} \sin \alpha=0 .
$$

After simplification of (5.26) we obtain

$$
4 n_{2}(\cos \alpha)^{2}-2 n_{1} \cos \alpha-\left(n_{2}+n_{3}\right)=0 .
$$

One can satisfy (5.26) only if $\cos (2 \pi / n)$ is a rational number or a quadratic irrationality. It is well known from Galouis theory that this happens only when

$$
\begin{aligned}
& n \in\{3,4,5,6,8,10,12\} ; \\
& \cos (2 \pi / n) \in \mathbb{Q} \quad \text { if } n=3,4,6 \text {; } \\
& \cos (2 \pi / n) \in \mathbb{Q}(\sqrt{2}) \quad \text { if } \quad n=8 \text {; } \\
& \cos (2 \pi / n) \in \mathbb{Q}(\sqrt{3}) \quad \text { if } \quad n=12 ; \\
& \cos (2 \pi / n) \in \mathbb{Q}(\sqrt{5}) \quad \text { if } \quad n=5,10 .
\end{aligned}
$$

All the quasicrystals having $n$-fold symmetry with $n$ not included in (5.28) do not satisfy strong LR.

Remark. Since different grids in (4.2) cannot be parallel, no quasicrystals correspond to $n=6$ and $n=10$ in (5.28). However, 6-fold symmetric and 10-fold symmetric quasicrystals can be constructed by an appropriate choice of the parameters $c_{i}$ in (4.2), where the grid functions are given by (5.24) with $n=3$ and $n=5$ respectively (the 10 -fold symmetric quasicrystal obtained in such a way is the Penrose tiling).

Periodic quasicrystals having $n=3,4,6$ obviously satisfy strong LR. The existence of strong LR for $n=10$ was shown by de Bruijn. Evidence for the absence of strong LR in the $n=8$-case was given by Beenker. Nothing is known about the $n=12$-case.

Let us try to express the restrictions of Theorems 1,2 to the two-dimensional quasicrystals in a more explicit form. Suppose that a quasicrystal given by the grids (4.2) satisfies strong LR.

Consider first the situation, when the number $n$ of grids is equal to three: $n=3$. This is the case of codimension one and hence Theorem 1 is applicable. We conclude that the quasicrystal is periodic. 
Now we study the case $n=4$ when there are four linear functions $f_{i}$ in (4.2). Since different grids are not parallel (by the definition of the dual graph) every two of the functions $f_{i}$ form a basis of $\left(\mathbb{R}^{2}\right)^{\prime}$. Choose $f_{1}$ and $f_{2}$ as such a basis and express $f_{3}$ and $f_{4}$ as linear combinations of $f_{1}, f_{2}$ :

$$
f_{3}=a f_{1}+b f_{2}, \quad f_{4}=c f_{1}+d f_{2} .
$$

We make use of Theorem 2 and apply the SI-conditions to all triplets of functions $f_{i}(i=1, \ldots, 4)$. For the four triplets of $f_{i}$ we introduce four triplets of integers

$$
\left(m_{1}, m_{2}, m_{3}\right), \quad\left(n_{1}, n_{2}, n_{3}\right), \quad\left(p_{1}, p_{2}, p_{3}\right), \quad\left(q_{1}, q_{2}, q_{3}\right)
$$

which appear in (5.1) and write the SI-conditions in the form (5.3) using the representation (5.29):

$$
\begin{aligned}
& \operatorname{det}\left\|\begin{array}{lll}
1 & 0 & m_{1} \\
0 & 1 & m_{2} \\
a & b & m_{3}
\end{array}\right\|=0, \quad \operatorname{det}\left\|\begin{array}{lll}
1 & 0 & n_{1} \\
0 & 1 & n_{2} \\
c & d & n_{3}
\end{array}\right\|=0, \\
& \operatorname{det}\left\|\begin{array}{lll}
1 & 0 & p_{1} \\
a & b & p_{2} \\
c & d & p_{3}
\end{array}\right\|=0, \quad \operatorname{det}\left\|\begin{array}{lll}
0 & 1 & q_{1} \\
a & b & q_{2} \\
c & d & q_{3}
\end{array}\right\|=0 .
\end{aligned}
$$

We consider the SI-conditions (5.30) as equations for unknown variables $a, b, c$, $d$. Since the number of equations is equal to the number of variables, Eqs. (5.30) can be solved (if they are independent). The properties of the solutions of Eqs. (5.30) are described in the following proposition.

\section{Proposition 5.}

1. If Eqs. (5.30) are independent, the solutions $a, b, c, d$ are either rational numbers or quadratic irrationalities:

$$
a, b, c, d \in \mathbb{Q}(\sqrt{D}) \text { for some integer } D .
$$

The number of different solutions of Eqs. (5.30) is equal to

a) one, if $a, b, c, d \in \mathbb{Q}$;

b) two, if $a, b, c, d \in \mathbb{Q}(\sqrt{D})$.

In the case b) the solutions are mutually conjugated algebraic numbers (the numbers $x, x^{\prime} \in \mathbb{Q}(\sqrt{D})$ are called conjugated if $x=a+b \sqrt{D}, x^{\prime}=a-b \sqrt{D}$, where $a, b \in \mathbb{Q})$.

2. If Eqs. (5.30) are not independent, then after a proper permutation of the functions $f_{1}, f_{2}, f_{3}, f_{4}$ is made the solutions $a, b, c, d$ are given $b y$

$$
\begin{aligned}
& a=a^{\prime}+a^{\prime \prime} t, \quad b=b^{\prime}+b^{\prime \prime} t, \\
& c=\frac{c^{\prime}+c^{\prime \prime} t}{h^{\prime}+h^{\prime \prime} t}, \quad d=\frac{d^{\prime}+d^{\prime \prime} t}{h^{\prime}+h^{\prime \prime} t},
\end{aligned}
$$

where $a^{\prime}, a^{\prime \prime}, b^{\prime}, b^{\prime \prime}, c^{\prime}, c^{\prime \prime}, d^{\prime}, d^{\prime \prime}, h^{\prime}, h^{\prime \prime} \in \mathbb{Q}$ and $t$ is a real parameter. Only eight of ten rational constants $a^{\prime}, \ldots, h^{\prime \prime}$ are independent. 
Proof. Since the triplets of integers $m_{j}, n_{j}, p_{j}, q_{j}(j=1,2,3)$ in (5.30) are related to four grids they have some special properties. Consider the triplet $\left(m_{1}, m_{2}, m_{3}\right)$. One checks easily that if two of $m_{j}(j=1,2,3)$ are equal to zero then the third $m_{j}$ equals zero too. The same is valid for $n_{j}, p_{j}, q_{j}(j=1,2,3)$. One can use this property and see that by a proper permutation of the functions $f_{1}, f_{2}, f_{3}, f_{4}$ in (5.29) Eqs. (5.30) are reduced to equations of the same type with an extra condition,

$$
m_{2} \neq 0, \quad n_{2} \neq 0 .
$$

after writing (5.30) explicitly we get

(i) $m_{3}-m_{1} a-m_{2} b=0$,

(ii) $n_{3}-n_{1} c-n_{2} d=0$,

(iii) $p_{3} b-p_{2} d+p_{1}(a d-b c)=0$,

(iv) $q_{2} c-q_{3} a+q_{1}(a d-b c)=0$.

By excluding the variables $b, d, c$ successively from Eqs. (5.34) one obtains (5.31) or (5.32).

Consider the case $n>4$.

Proposition 6. Let the SI-condition be satisfied for every triplet of the grid functions $f_{i}$.

1. If there exist four functions $f_{j}, f_{k}, f_{l}, f_{m}$ such that Eqs. (5.20) for them are independent, then one can express all functions $f_{i}$ as linear combinations of two, e.g. $f_{j}$ and $f_{k}$, with the coefficients being quadratic irrationalities:

$$
\begin{gathered}
f_{i}(\mathbf{x})=a_{i} f_{j}(\mathbf{x})+b_{i} f_{k}(\mathbf{x}), \quad \mathbf{x} \in \mathbb{R}^{2}, \\
a_{i}, b_{i} \in \mathbb{Q}(\sqrt{D}), \quad D \in \mathbb{Z} .
\end{gathered}
$$

2. If for every four functions $f_{j}, f_{k}, f_{l}, f_{m}$ Eqs. (5.30) are not independent, then one can express all the functions $f_{i}$ as linear combinations of two, say $f_{1}$ and $f_{2}$, as

$$
\begin{aligned}
f_{i}(\mathbf{x}) & =a_{i} f_{1}(\mathbf{x})+b_{i} f_{2}(\mathbf{x}), \quad \mathbf{x} \in \mathbb{R}^{2}, \\
a_{i} & =\frac{a_{i}^{\prime}+a_{i}^{\prime \prime} t}{h_{i}^{\prime}+h_{i}^{\prime \prime} t}, \quad b_{i}=\frac{b_{i}^{\prime}+b_{i}^{\prime \prime} t}{h_{i}^{\prime}+h_{i}^{\prime \prime} t},
\end{aligned}
$$

where $t$ is a real parameter, $a_{i}^{\prime}, a_{i}^{\prime \prime}, b_{i}^{\prime}, b_{i}^{\prime \prime}, h_{i}^{\prime}, h_{i}^{\prime \prime} \in \mathbb{Q}$ for all $i$. Not all of the constants $a_{i}^{\prime}, \ldots, h_{i}^{\prime \prime}$ are independent.

The proof of this proposition can be obtained immediately by making use of Proposition 5.

We see that the limitations for the quasicrystals satisfying strong LR derived in Theorem 2 are essentially reduced to (5.35) or, in some exceptional cases, to (5.36). One should note that the set (5.36) of "exceptional" quasicrystals is non-countable, whereas the "regular" set (5.35) is countable. It was mentioned in Sect. 1 that the set of restorable quasicrystals is countable. We suspect that the quasicrystals (5.36) do not have strong LR although we have not proved this. 


\section{Quasicrystals Based on Quadratic Irrationalities}

In this section we study the quasicrystals generated by quadratic irrationalities. Let us repeat the definition (1.6) in a slightly different form. For the $v$-quasicrystal associated with the embedding (1.3) we consider the orthogonal complement $v^{*}$ to the subspace $\operatorname{Im}(v)$ of $\mathbb{R}^{2}$.

Definition. We say that the $v$-quasicrystal is based on quadratic irrationalities of the field $\mathbb{Q}(\sqrt{D})$ if $v^{*}$ is spanned by a set of vectors $\left\{\mathbf{g}_{j}\right\}\left(\mathbf{g}_{j} \in \mathbb{R}^{n}, j=1, \ldots, n-2\right)$ of the form

$$
\mathbf{g}=\left(y_{1}, \ldots, y_{n}\right), \quad \text { where } y_{i} \in \mathbb{Q}(\sqrt{D}) .
$$

In terms of grid functions $f_{i}$ this definition means that every function $f_{j}(j=3, \ldots, n)$ can be expressed as a linear combination of $f_{1}, f_{2}$ with the coefficients belonging to $\mathbb{Q}(\sqrt{D})$ :

$$
f_{j}(\mathbf{x})=a_{j} f_{1}(\mathbf{x})+b_{j} f_{2}(\mathbf{x}),
$$

where $j=3, \ldots, n, \mathbf{x} \in \mathbb{R}^{2}, a_{j}, b_{j} \in \mathbb{Q}(\sqrt{D})$.

The principal result of this section is that a wide class of quasicrystals (6.2) has weak LR (see Theorem 3 below). We start with a very simple assertion.

Lemma 9. If a quasicrystal (4.2) is based on quadratic irrationalities, then every triplet $\left(f_{j}, f_{k}, f_{l}\right)$ satisfies the SI-condition.

One obtains the proof easily by substituting $f_{i}(\mathbf{x})=a_{i} f_{1}(\mathbf{x})+b_{i} f_{2}(\mathbf{x})$ with $a_{i}=a_{i}^{\prime}+a_{i}^{\prime \prime} \sqrt{D}, b_{i}=b_{i}^{\prime}+b_{i}^{\prime \prime} \sqrt{D}\left(a_{i}^{\prime}, a_{i}^{\prime \prime}, b_{i}^{\prime}, b_{i}^{\prime \prime} \in \mathbb{Q}\right)$ in the SI-conditions (5.3).

We introduce now some constructions which will be used in the proof of Theorem 3. Consider an arbitrary quasicrystal (1.3) and find its $r$-atlas for some positive $r$. Take this $r$-atlas as local rules. Let some tiling of the plane $\mathbb{R}^{2}$ satisfy these rules. One can expect that when $r$ is sufficiently large this tiling resembles some properties of the quasicrystal. Particularly we show below that the lifting of the tiling goes almost in the same direction as the lifting of the quasicrystal.

Consider the dual graph of the tiling and that of the quasicrystal. We perform a continuous transformation $\mathbb{R}^{2} \rightarrow \mathbb{R}^{2}$ such that the first and the second array of curves of the dual graph are transformed onto two grids

$$
x_{j}=k_{j}, \quad\left(j=1,2, k_{j} \in \mathbb{Z}\right) .
$$

Other arrays of curves of the dual graph are not necessarily transformed onto grids by the transformation. Find a linear transformation $\mathbb{R}^{2} \rightarrow \mathbb{R}^{2}$ such that the first two grids of the quasicrystal are transformed onto the grids (6.3). Since the transformation is linear, the other grids of the dual graph are transformed onto some grids

$$
f_{j}(\mathbf{x})=k_{j}+c_{j}, \quad\left(j=3, \ldots, n, \mathbf{x} \in \mathbb{R}^{2}, k_{j} \in \mathbb{Z}, c_{j} \in \mathbb{R}\right) .
$$

Take $j \in\{3, \ldots, n\}$ and consider an arbitrary curve of the $j^{\text {th }}$ array of the dual graph of the tiling (after the transformation is performed). Although this curve is not necessarily straight its slope is close to the slope of the $j^{\text {th }}$ grid. To explain what we mean let us consider two points $\mathbf{x}_{1}, \mathbf{x}_{2}$ on this curve which are contained in a map of the tiling having radius less than $r$. Since the $r$-atlas of the tiling is included in the $r$-atlas of the quasicrystal one can find two new points $\mathbf{x}_{1}^{\prime}, \mathbf{x}_{2}^{\prime}$ such that 
a) the point $\mathbf{x}_{m}^{\prime}$ belongs to the same square of the lattice formed by the grids (6.3) as the point $\mathbf{x}_{m}(m=1,2)$;

b) the segment $\left(\mathbf{x}_{1}^{\prime}, \mathbf{x}_{2}^{\prime}\right)$ is parallel to the lines of the $j^{\text {th }}$ grid of the quasicrystal.

The maximal distance between the points $\mathbf{x}_{1}, \mathbf{x}_{2}$ is of order $r$ because they are included in a map of the $r$-atlas. We conclude that the slope of the curve differs from that of the $j^{\text {th }}$ grid to not more than const $/ r$. We need this result reformulated in terms of lifting functions.

Lemma 10. Let a tiling satisfy the r-rules given by the r-atlas of a quasicrystal. Apply the introduced continuous transformations $\mathbb{R}^{2} \rightarrow \mathbb{R}^{2}$ to the dual graphs of the tiling and of the quasicrystal as explained above. Consider the lifting of the transformed dual graph of the tiling

$$
w(\mathbf{x})=\left(\left[x_{1}\right],\left[x_{2}\right], w_{3}(\mathbf{x}), \ldots, w_{n}(\mathbf{x})\right), \quad\left(\mathbf{x} \in \mathbb{R}^{2}\right)
$$

and that of the grid pattern

$$
w^{\prime}(\mathbf{x})=\left(\left[x_{1}\right],\left[x_{2}\right],\left[f_{3}(\mathbf{x})\right], \ldots,\left[f_{n}(\mathbf{x})\right]\right), \quad\left(\mathbf{x} \in \mathbb{R}^{2}\right) .
$$

There exist two constants $c_{1}>0, c_{2}>0$ such that

$$
\left|\left(w_{i}(\mathbf{y})-w_{i}\left(\mathbf{y}^{\prime}\right)\right)-\left(f_{i}(\mathbf{y})-f_{i}\left(\mathbf{y}^{\prime}\right)\right)\right|<c_{1}+\left(c_{2} / r\right)\left\|\mathbf{y}-\mathbf{y}^{\prime}\right\|
$$

for all $i$ and $\mathbf{y}, \mathbf{y}^{\prime} \in \mathbb{R}^{2}$.

The assertion of Lemma 10 is valid for an arbitrary quasicrystal. We show below that for a broad class of quasicrystals (6.1), (6.2) the parameter $c_{2}$ in (6.7) is equal to zero. This means that the lifting $w$ of the tiling differs from the lifting $w^{\prime}$ of the quasicrystal to not more than a constant, i.e. the tiling satisfies weak LR.

Theorem 3. Let a two-dimensional quasicrystal with $n=4$ be such that

a) every triplet of the grid functions $f_{i}$ satisfies the SI-conditions (5.3);

b) four equations (5.30) are independent.

If some auxiliary constraints for the integer numbers $m_{j}, n_{j}, p_{j}, q_{j}(j=1,2,3)$ are satisfied (see (6.19) and (6.34)), then the quasicrystal satisfies weak LR.

The auxiliary constraints mentioned in Theorem 3 mean nothing more than all the expressions which will appear in the denominators of different fractions during the proof are assumed to take nonzero values. Since these constraints are not very important but rather cumbersome we analyze them at the end of the proof.

First we discuss the idea of the following proof on a nonrigorous level. Choose some positive $r$ and take the $r$-atlas of the quasicrystal as LR. If $r$ is sufficiently large the lifting functions $w_{i}(6.5)$ are close to $f_{i}(6.6)$ in the sense of Lemma 10. We neglect for some time by the discontinuities of the lifting functions $w_{i}$ and consider them as smooth functions at scales larger than the separations of the lines in the dual graph. Suppose further that linearizations (linear parts) of $w_{i}$ approximate them well at sufficiently large scales. These linearizations satisfy the SI-condition (5.3) everywhere the plane. Since Eqs. (5.30) are independent, they have only two solutions. So, we can use Proposition 5 and conclude that all derivatives $\frac{\partial w_{i}}{\partial x_{k}}$ are constant on the whole plane. This means in turn that $w_{i}$ are linear functions corresponding to one of the solutions of Eqs. (5.30) (see Proposition 5). One can exclude one of these 
solutions by choosing sufficiently large $r$ and making use of Lemma 10: according to Lemma 10 all functions $w_{i}$ are close to $f_{i}$ when $r$ is large. Combining the above results we obtain that $w_{i}$ are identical to $f_{i}$ in (6.6). The reality is that the functions $w_{i}$ are not continuous (and non-differentiable as well!). Nevertheless, the rigorous proof of Theorem 3 is based on the same ideas.

\section{Proof of the Main Theorem 3}

Step 1 (SI-Conditions for Dual Graphs). Let us choose LR being identical to the $r$-atlas of the quasicrystal for some large $r$. The exact value of $r$ will be determined at the end of the proof.

Let a tiling of the plane satisfy these rules. Find a continuous transformation $\mathbb{R}^{2} \rightarrow \mathbb{R}^{2}$ which transforms the first two arrays of curves belonging to the dual graph of the tiling onto the grids (6.3), and a linear transformation $\mathbb{R}^{2} \rightarrow \mathbb{R}^{2}$ which transforms the first two grids of the quasicrystal onto the grids (6.3), as explained before Lemma 10. The corresponding lifting functions $w, w^{\prime}$ have the form (6.5), (6.6). Since SI-conditions are satisfied for the grids of the quasicrystal, the functions $f_{3}$ and $f_{4}$ are given by

$$
\begin{aligned}
& f_{3}(\mathbf{x})=a x_{1}+b x_{2}, \\
& f_{4}(\mathbf{x})=c x_{1}+d x_{2},
\end{aligned}
$$

where $a, b, c, d$ are solutions of (5.30). In order to prove the existence of weak LR we must show that for sufficiently large $r$ two functions

$$
w_{j}(\mathbf{x})-f_{j}(\mathbf{x}) \quad(j=3,4)
$$

are bounded on the whole plane.

We need to modify the definition of SI-conditions in order to make it applicable for arbitrary dual graphs [not only for grids as in (5.1)]. Let $w(\mathbf{x})=\left(w_{1}(\mathbf{x}), \ldots, w_{n}(\mathbf{x})\right)$ be the lifting function of a dual graph. Let $\left(w_{j}(\mathbf{x}), w_{k}(\mathbf{x}), w_{l}(\mathbf{x})\right)$ be a triplet of the component functions of the lifting $w(\mathbf{x})(j, k, l \in\{1,2,3,4\})$.

Definition. The SI-condition is satisfied for the triplet $\left(w_{j}, w_{k}, w_{l}\right)$ and three integers $m_{1}, m_{2}, m_{3}$ if for any $\mathbf{x} \in \mathbb{R}^{2}$ one can find $\mathbf{x}^{\prime} \in \mathbb{R}^{2}$ such that $w_{j}\left(\mathbf{x}^{\prime}\right)=w_{j}(\mathbf{x})+m_{1}, w_{k}\left(\mathbf{x}^{\prime}\right)$ $=w_{k}(\mathbf{x})+m_{2}, w_{l}\left(\mathbf{x}^{\prime}\right)=w_{l}(\mathbf{x})+m_{3}$.

It is clear that the definition of SI-condition for grids is a particular case of the more general definition given above. One proves an easy

Lemma 11. If the radius $r$ of the local rules defined above is sufficiently large then the tiling satisfies the SI-conditions identical to that of the quasicrystal (with the same triplets of integers).

One can write these conditions for the component functions $w_{i}$ of the lifting $w$. Consider the triplet $\left(w_{1}, w_{2}, w_{3}\right)$ as an example. The SI-condition for this triplet states that for every point $\mathbf{x}$ of the plane there exists a point $\mathbf{x}^{\prime}$ such that

$$
\begin{aligned}
& w_{1}\left(\mathbf{x}^{\prime}\right)=w_{1}(\mathbf{x})+m_{1}, \\
& w_{2}\left(\mathbf{x}^{\prime}\right)=w_{2}(\mathbf{x})+m_{2}, \\
& w_{3}\left(\mathbf{x}^{\prime}\right)=w_{3}(\mathbf{x})+m_{3} .
\end{aligned}
$$


If we use the so-obtained point $\mathbf{x}^{\prime}$ instead of $\mathbf{x}$ in the SI-conditions, then we find another point $\mathbf{x}^{\prime \prime}$ such that

$$
\begin{aligned}
& w_{1}\left(\mathbf{x}^{\prime \prime}\right)=w_{1}\left(\mathbf{x}^{\prime}\right)+m_{1}, \\
& w_{2}\left(\mathbf{x}^{\prime \prime}\right)=w_{2}\left(\mathbf{x}^{\prime}\right)+m_{2}, \\
& w_{3}\left(\mathbf{x}^{\prime \prime}\right)=w_{3}\left(\mathbf{x}^{\prime}\right)+m_{3} .
\end{aligned}
$$

Since this procedure can be repeated any finite number of times, we conclude that for any integer $k$ and every point $\mathbf{x}$ of the plane there exists a point $\mathbf{x}^{\prime}$ such that

$$
\begin{aligned}
& w_{1}\left(\mathbf{x}^{\prime}\right)=w_{1}(\mathbf{x})+k m_{1}, \\
& w_{2}\left(\mathbf{x}^{\prime}\right)=w_{2}(\mathbf{x})+k m_{2}, \\
& w_{3}\left(\mathbf{x}^{\prime}\right)=w_{3}(\mathbf{x})+k m_{3} .
\end{aligned}
$$

The SI-conditions for other triplets of $w_{i}$ are quite analogous to (6.11). Recall that after the transformations $\mathbb{R}^{2} \rightarrow \mathbb{R}^{2}$ found above are performed, the lifting function takes the form (6.5). Let us rewrite the SI-conditions (6.11) for this particular case.

Step 2 ( Triplets $(1,2,3),(1,2,4))$. The SI-condition for the triplet $\left(w_{1}, w_{2}, w_{3}\right)$ states that for any integer $k$ and every point $\mathbf{x}=\left(x_{1}, x_{2}\right)$ of the plane there exists a point $\mathbf{x}^{\prime}=\left(x_{1}^{\prime}, x_{2}^{\prime}\right)$ such that

$$
\begin{gathered}
x_{1}^{\prime}=x_{1}+k m_{1}, \\
x_{2}^{\prime}=x_{2}+k m_{2}, \\
w_{3}\left(\mathbf{x}^{\prime}\right)=w_{3}(\mathbf{x})+k m_{3} .
\end{gathered}
$$

We know from Lemma 10 that the functions $w_{3}$ and $w_{4}$ are close to $a x_{1}+b x_{2}$ and $c x_{1}+d x_{2}$ respectively if $r$ is large enough. Bearing this in mind we introduce new functions $F$ and $\Phi$ such that

$$
\begin{aligned}
& w_{3}(\mathbf{x})=F(\mathbf{x})+a x_{1}+b x_{2}, \\
& w_{4}(\mathbf{x})=\Phi(\mathbf{x})+c x_{1}+d x_{2}, \quad \mathbf{x}=\left(x_{1}, x_{2}\right) \in \mathbb{R}^{2} .
\end{aligned}
$$

$F(\mathbf{x})$ and $\Phi(\mathbf{x})$ are exactly the functions (6.9) and we have to prove that both $F(\mathbf{x})$ and $\Phi(\mathbf{x})$ are uniformly bounded on $\mathbb{R}^{2}$. Condition (6.12) reads:

$$
F\left(x_{1}+k m_{1}, x_{2}+k m_{2}\right)=F\left(x_{1}, x_{2}\right) \text { for all integer } k .
$$

This means that $F(\mathbf{x})$ mainly depends on the variable

$$
u=m_{2} x_{1}-m_{1} x_{2}
$$

only, or, more precisely, that a function $f: \mathbb{R} \rightarrow \mathbb{R}$ exists such that the difference $F(\mathbf{x})-f(u(\mathbf{x}))$ is bounded:

$$
|F(\mathbf{x})-f(u(\mathbf{x}))|<\text { const for all } \mathbf{x} \in \mathbb{R}^{2} .
$$

The function $u(\mathbf{x})$ in $(6.16)$ is given by (6.15).

Remark. Since we wish to prove that $F(\mathbf{x})$ and $\Phi(\mathbf{x})$ are bounded, we shall neglect all bounded functions everywhere below and write $A(x)=B(x)$ instead of " $|A(\mathbf{x})-B(\mathbf{x})|<$ const for all $\mathbf{x} \in \mathbb{R}^{2}$." 
In accordance with this convention we write (6.16) as

$$
F(\mathbf{x})=f(u(\mathbf{x})),
$$

where $u(\mathbf{x})=m_{2} x_{1}-m_{1} x_{2}, \mathbf{x}=\left(x_{1}, x_{2}\right) \in \mathbb{R}^{2}$.

The analysis of the SI-condition for the triplet $\left(w_{1}, w_{2}, w_{4}\right)$ can be performed in a quite similar way. The result is that a function $\varphi: \mathbb{R} \rightarrow \mathbb{R}$ exist such that

$$
\Phi(\mathbf{x})=\varphi(v(\mathbf{x})),
$$

where $v(\mathbf{x})=n_{2} x_{1}-n_{1} x_{2}, \mathbf{x}=\left(x_{1}, x_{2}\right) \in \mathbb{R}^{2}$. We obtain that both $F(\mathbf{x})$ and $\Phi(\mathbf{x})$ depend on one variable (the corresponding variables are $u$ and $v$ respectively).

Suppose that two functions $u(\mathbf{x})$ and $v(\mathbf{x})$ are independent, i.e.

$$
\Delta=\operatorname{det}\left\|\begin{array}{ll}
m_{1} & m_{2} \\
n_{1} & n_{2}
\end{array}\right\| \neq 0 .
$$

It is convenient to choose $u$ and $v$ as new coordinates instead of $x_{1}, x_{2}$. The variables $x_{1}$ and $x_{2}$, as functions of $u$ and $v$, are given by

$$
x_{1}=\left(m_{1} v-n_{1} u\right) / \Delta, \quad x_{2}=\left(m_{2} v-n_{2} u\right) / \Delta .
$$

Step 3 (Triplets $(1,3,4),(2,3,4)$ ). Consider the SI-condition for the triplet $\left(w_{1}, w_{3}, w_{4}\right)$. It states that for any integer $k$ and every point $\mathbf{x} \in \mathbb{R}^{2}$ one can find a point $\mathbf{x}^{\prime} \in \mathbb{R}^{2}$ such that

$$
\begin{aligned}
& w_{1}\left(\mathbf{x}^{\prime}\right)=w_{1}(\mathbf{x})+k p_{1}, \\
& w_{3}\left(\mathbf{x}^{\prime}\right)=w_{3}(\mathbf{x})+k p_{2}, \\
& w_{4}\left(\mathbf{x}^{\prime}\right)=w_{4}(\mathbf{x})+k p_{3} .
\end{aligned}
$$

Using (6.13), (6.17), (6.18) and taking into account that $w_{1}(\mathbf{x})=x_{1}$ we rewrite (6.21) in new variables $u, v$. Condition (6.21) means that for any integer $k$ and a point $(u, v)$ of $\mathbb{R}^{2}$ there exists a point $\left(u+u^{\prime}, v+v^{\prime}\right)$ such that

$$
\begin{gathered}
m_{1} v^{\prime}-n_{1} u^{\prime}=\Delta p_{1} k, \\
f\left(u+u^{\prime}\right)-f(u)=\left(p_{2}-a p_{1}\right) k-b\left(m_{2} v^{\prime}-n_{2} u^{\prime}\right) / \Delta, \\
\varphi\left(v+v^{\prime}\right)-\varphi(v)=\left(p_{3}-c p_{1}\right) k-d\left(m_{2} v^{\prime}-n_{2} u^{\prime}\right) / \Delta .
\end{gathered}
$$

By making use of (5.34) we exclude $u^{\prime}$ and $v^{\prime}$ from the right-hand side of (6.22) and get

(i) $d\left(f\left(u+u^{\prime}\right)-f(u)\right)=b\left(\varphi\left(v+v^{\prime}\right)-\varphi(v)\right)$,

(ii) $b\left(m_{2} v^{\prime}-n_{2} u^{\prime}\right) / \Delta=\left(p_{2}-a p_{1}\right) k-\left(f\left(u+u^{\prime}\right)-f(u)\right)$,

(iii) $m_{1} v^{\prime}-n_{1} u^{\prime}=\Delta p_{1} k$.

Suppose that $b \neq 0$. If this is the case, one can use (6.23.ii), (6.23.iii) to express $u^{\prime}$ and $v^{\prime}$ as functions of $k$ and $f\left(u+u^{\prime}\right)-f(u)$ :

(i) $d\left(f\left(u+u^{\prime}\right)-f(u)\right)=b\left(\varphi\left(v+v^{\prime}\right)-\varphi(v)\right)$,

(ii) $u^{\prime}=A k+A^{\prime}\left(f\left(u+u^{\prime}\right)-f(u)\right)$,

(iii) $v^{\prime}=B k+B^{\prime}\left(f\left(u+u^{\prime}\right)-f(u)\right)$, 
where

$$
\begin{gathered}
A=\left(p_{1} m_{3}-p_{2} m_{1}\right) / b, \quad A^{\prime}=m_{1} / b, \\
B=\left(p_{1}\left(n_{1} a+n_{2} b\right)-p_{2} n_{1}\right) / b, \quad B^{\prime}=n_{1} / b .
\end{gathered}
$$

The SI-condition for the triplet $\left(w_{1}, w_{3}, w_{4}\right)$ says that for all $(u, v) \in \mathbb{R}^{2}$ and $k \in \mathbb{Z}$, Eqs. (6.24) can be satisfied simultaneously by properly chosen $u^{\prime}, v^{\prime}$. The thusobtained form (6.24) of the SI-condition for the triplet $\left(w_{1}, w_{3}, w_{4}\right)$ is the most convenient one for our analysis.

After similar calculations for the triplet $\left(w_{2}, w_{3}, w_{4}\right)$ we obtain the SI-condition rewritten in new variables $u, v$. The condition gives for any $k \in \mathbb{Z}$ and arbitrary point $(u, v) \in \mathbb{R}^{2}$ the existence of a point $\left(u+u^{\prime}, v+v^{\prime}\right) \in \mathbb{R}^{2}$ such that

(i) $c\left(f\left(u+u^{\prime}\right)-f(u)\right)=a\left(\varphi\left(v+v^{\prime}\right)-\varphi(v)\right)$,

(ii) $u^{\prime}=F k+F^{\prime}\left(f\left(u+u^{\prime}\right)-f(u)\right)$,

(iii) $v^{\prime}=G k+G^{\prime}\left(f\left(u+u^{\prime}\right)-f(u)\right)$,

where

$$
\begin{gathered}
F=\left(q_{2} m_{2}-q_{1} m_{3}\right) / a, \quad F^{\prime}=-m_{2} / a, \\
G=\left(n_{2} q_{2}-q_{1}\left(n_{1} a+n_{2} b\right)\right) / a, \quad G^{\prime}=-n_{2} / a .
\end{gathered}
$$

Step 4

Remark. In what follows we show that if the function $f(u)$ is not bounded then SIconditions (6.24) and (6.25) contradict each other. There are two difficulties with Eqs. (6.24), (6.25) which are in the way of establishing the contradiction:

1) $u^{\prime}$ appears not only in the left-hand side of Eqs. (6.24), (6.25) but also in the right-hand side as a part of the argument of function $f$. This makes it difficult to give a good estimate of $f\left(u+u^{\prime}\right)+f(u)$.

2) Not only $f$ but also $\varphi$ takes part in Eqs. (6.24), (6.25). So one has to eliminate $\varphi$.

We prove a useful lemma which shows how one can avoid difficulty 1.

Lemma 12. Let a function $f: \mathbb{R} \rightarrow \mathbb{R}$ satisfy the modified Lipshitz condition

$$
\left|f\left(u+u^{\prime}\right)-f(u)\right|<c+c^{\prime}\left|u^{\prime}\right|
$$

with positive $c$ and $c^{\prime}$ for all $u, u^{\prime}$. Let two constants $A, A^{\prime}$ such that $\left|c^{\prime} A^{\prime}\right|<1$ be specified. Then there exist positive constants $C, C^{\prime}$ such that

$$
\begin{aligned}
& \text { if } u^{\prime}=A+A^{\prime}\left(f\left(u+u^{\prime}\right)-f(u)\right) \text { for some } u, u^{\prime} \in \mathbb{R} \text {, } \\
& \text { then }\left|f\left(u+u^{\prime}\right)-f(u)\right|<C+C^{\prime}|f(u+A)-f(u)| .
\end{aligned}
$$

Proof.

$$
\begin{aligned}
\left|f\left(u+u^{\prime}\right)-f(u)\right| & <\left|f\left(u+u^{\prime}\right)-f(u+A)\right|+|f(u+A)-f(u)| \\
& <c+c^{\prime}\left|u^{\prime}-A\right|+|f(u+A)-f(u)| \\
& <c+\left|c^{\prime} A^{\prime}\right|\left|f\left(u+u^{\prime}\right)-f(u)\right|+|f(u+A)-f(u)| .
\end{aligned}
$$


We obtain (6.27) with

$$
C=c /\left(1-\left|c^{\prime} A^{\prime}\right|\right), \quad C^{\prime}=1 /\left(1-\left|c^{\prime} A^{\prime}\right|\right) .
$$

Consequences of Lemma 12. One can find positive constants $C, C^{\prime}$ such that

1) if for some $u, u^{\prime}, v, v^{\prime} \in \mathbb{R}$ and $k \in \mathbb{Z}$, Eqs. (6.24) are satisfied, then

$$
\left|f\left(u+u^{\prime}\right)-f(u)\right|<C+\left(C^{\prime} / r\right)|f(u+A k)-f(u)|
$$

2) if for some $u, u^{\prime}, v, v^{\prime} \in \mathbb{R}$ and $k \in \mathbb{Z}$, Eqs. (6.25) are satisfied, then

$$
\left|f\left(u+u^{\prime}\right)-f(u)\right|<C+\left(C^{\prime} / r\right)|f(u+F k)-f(u)| .
$$

Proof. According to Lemma 10 the function $f(u)$ satisfies the modified Lipshitz condition (6.26) with $c^{\prime}=$ const $/ r$. Therefore, after applying Lemma 12 to (6.24), (6.25) we get (6.28), (6.29), respectively.

\section{Step 5 (Elimination of $\varphi$ )}

Remark. Up to now we considered Eqs. (6.24) [and Eqs. (6.25)] as SI-conditions: whenever $u, v, k$ are given they define $u^{\prime}$ and $v^{\prime}$. One can try to view Eqs. (6.24) from another side: for all $u, v, v^{\prime}$ they define $u^{\prime}$ and $k$. Really, if we know $u, v, v^{\prime}$, then we can find $f\left(u+u^{\prime}\right)-f(u)$ from (6.24.i) (assuming that $d \neq 0$ ), then obtain $k$ from Eq. (6.24.iii) (assuming that $B \neq 0$ ), and finally get $u^{\prime}$ from Eq. (6.24.ii) (the same concerns Eqs. (6.25) provided $c \neq 0, G \neq 0$ ).

To eliminate $\varphi$ we have to consider Eqs. (6.24), (6.25) simultaneously. Let us use $\bar{u}, \bar{v}, \bar{u}^{\prime}, \bar{v}^{\prime}, \bar{k}$ to denote the variables of (6.25) and reserve the old notation $u, v, u^{\prime}, v^{\prime}, k$ for the variables of (6.24). Let independent variables in (6.25) be $\bar{u}, \bar{v}, \bar{v}^{\prime}$ according to the above Remark [for Eqs. (6.24) we use the standard triplet $u, v, k$ of independent variables]. Equations (6.24), (6.25) enable one to determine $u^{\prime}, v^{\prime}, \bar{k}, \bar{u}^{\prime}$ whenever $u, v$, $k, \bar{u}, \bar{v}, \bar{v}^{\prime}$ are known.

Let us choose some $u, v, k$, and $\bar{u}$, then find $u^{\prime}, v^{\prime}$ from (6.24) and put

$$
\bar{v}=v, \quad \bar{v}^{\prime}=v^{\prime} .
$$

This means that instead of six independent variables $u, v, k, \bar{u}, \bar{v}, \bar{v}^{\prime}$ we consider only four $u, v, k, \bar{u}$, while $\bar{v}$ and $\bar{v}^{\prime}$ depend on them according to (6.30). Relations (6.30) make it possible to exclude $v, \bar{v}, v^{\prime}, \bar{v}^{\prime}$ from $(6.24),(6.25)$ and get

(i) $a d\left(f\left(u+u^{\prime}\right)-f(u)\right)=b c\left(f\left(\bar{u}+\bar{u}^{\prime}\right)-f(\bar{u})\right)$,

(ii) $u^{\prime}=A k+A^{\prime}\left(f\left(u+u^{\prime}\right)-f(u)\right)$,

(iii) $\bar{u}^{\prime}=F \bar{k}+F^{\prime}\left(f\left(\bar{u}+\bar{u}^{\prime}\right)-f(\bar{u})\right)$,

(iv) $B k+B^{\prime}\left(f\left(u+u^{\prime}\right)-f(u)\right)=G \bar{k}+G^{\prime}\left(f\left(\bar{u}+\bar{u}^{\prime}\right)-f(\bar{u})\right)$.

$\left[A, A^{\prime}, B, B^{\prime}, F, F^{\prime}, G, G^{\prime}\right.$ are defined in (6.24), (6.25).] Note that we still have four independent variables in (6.31), i.e. for all values of $u, v, k, \bar{u}$ there exist $u^{\prime}, \bar{u}^{\prime}, \bar{k}$ such that Eqs. (6.31) are satisfied. More precisely, $v$ does not participate in (6.31), so the number of independent variables is reduced to three, they are $u, \bar{u}, k$. So we are left with equations for the function $f$ only. 
Step 6 (Final). By applying consequences (6.28), (6.29) of Lemma 12 to (6.31.i) and using (6.31.ii), (6.31.iii), (6.31.iv) one can find positive constants $c, c^{\prime}$, and $c^{\prime \prime}$, such that

$$
\begin{aligned}
& |S(f(u+A k)-f(u))-T(f(\bar{u}+H k)-f(\bar{u}))|<c \\
& \quad+\frac{c^{\prime}}{r}|f(u+A k)-f(u)|+\frac{c^{\prime \prime}}{r}|f(\bar{u}+H k)-f(\bar{u})| \text { for all } u, \bar{u}, k,
\end{aligned}
$$

where $S=a d, T=b c, H=F(B / G)$. Inequality (6.32) enables one to prove that $f$ is bounded.

Lemma 13. Let function $f$ satisfy the modified Lipshitz condition (6.26) and inequality (6.32) with nonzero $A, H, S, T$. If $S A \neq T H$ in (6.32) and $r$ is sufficiently large, then $f$ is a bounded function.

Proof. Suppose that $f$ is not bounded. Choose a large number $M$ and find $x_{0} \in \mathbb{R}$, $k \in \mathbb{Z}$ such that $\left|f\left(x_{0}+H k\right)-f(x)\right|>M$. From (6.32) obtain that for all $x, y \in \mathbb{R}$,

(i) $\left|\frac{f(x+A k)-f(x)}{f(y+A k)-f(y)}-1\right|<\frac{C}{r}+\frac{C^{\prime}}{M}$,

(ii) $\left|\frac{f(x+H k)-f(x)}{f(y+H k)-f(y)}-1\right|<\frac{C}{r}+\frac{C^{\prime}}{M}$,

where $C$ and $C^{\prime}$ are some constants [first one has to prove (6.33.i) and then gets (6.33.ii) as a consequence of (6.32), (6.33.i)].

Consider two sequences $\left\{x_{j}\right\},\left\{y_{j}\right\}: x_{j}=x_{0}+H k j, y_{j}=x_{0}+A k j$. Define

$$
\begin{aligned}
X & =\inf _{j \neq m}\left[\left(f\left(x_{j}\right)-f\left(x_{m}\right)\right) /\left(x_{j}-x_{m}\right)\right], \\
X^{\prime} & =\sup _{j \neq m}\left[\left(f\left(x_{j}\right)-f\left(x_{m}\right)\right) /\left(x_{j}-x_{m}\right)\right], \\
Y & =\inf _{j \neq m}\left[\left(f\left(y_{j}\right)-f\left(y_{m}\right)\right) /\left(y_{j}-y_{m}\right)\right], \\
Y^{\prime} & =\sup _{j \neq m}\left[\left(f\left(y_{j}\right)-f\left(y_{m}\right)\right) /\left(y_{j}-y_{m}\right)\right] .
\end{aligned}
$$

If $S A \neq T H$ and both $r$ and $M$ is large enough, then (6.32) and (6.33) imply that the segments $\left[X, X^{\prime}\right]$ and $\left[Y, Y^{\prime}\right]$ do not intersect. This is in contradiction with (6.26), since for every $m \in \mathbb{Z}$ one can find $j \in \mathbb{Z}$ such that $\left|x_{j}-y_{m}\right|<\operatorname{const} k$. Lemma 13 is proven.

Thus we obtain that $f$ is bounded if

$$
\begin{gathered}
a \neq 0, \quad b \neq 0, \quad c \neq 0, \quad d \neq 0, \quad A \neq 0, \quad B \neq 0, \quad F \neq 0, \quad G \neq 0, \\
a d A G \neq b c B F .
\end{gathered}
$$

Since $f$ is bounded, $\varphi$ is bounded as well due to (6.24.i).

We established that Theorem 3 is valid provided (6.19) and (6.34).

Let us discuss an interesting example of an application of Theorem 3. Consider the two-dimensional quasicrystal having pentagonal symmetry. Its grid functions $f_{i}$ are given by

$$
f_{j}(\mathbf{x})=\cos \left(\frac{2 \pi}{5} j\right) x_{1}+\sin \left(\frac{2 \pi}{5} j\right) x_{2}, \quad\left(j=0, \ldots, 4 ; \mathbf{x} \in \mathbb{R}^{2}\right) .
$$


The dual graph consists of the grids

$$
f_{j}(\mathbf{x})=z_{j}+c_{j} \quad\left(z_{j} \in \mathbb{Z}\right) .
$$

If $c_{j}$ are such that $\gamma=\sum_{j} c_{j}=0$, we obtain Penrose tiling for which the existence of strong LR was established by de Bruijn. What happens when $\gamma \neq 0$ ?

Proposition 7 (consequence of Theorem 3). All 5-fold symmetric quasicrystals (6.35), (6.36) satisfy weak LR.

Proof. Let us erase one of the grids (6.36), say $f_{0}(\mathbf{x})=z_{0}+c_{0}$. One can try to apply Theorem 3 to the remaining grids given by $(6.35),(6.36)$ with $j=1,2,3,4$. Simple calculations show that

$$
f_{3}(\mathbf{x})=a f_{1}(\mathbf{x})+b f_{2}(\mathbf{x}), \quad f_{4}(\mathbf{x})=c f_{1}(\mathbf{x})+d f_{2}(\mathbf{x})
$$

where $a=-1, c=d=-b=(1-\sqrt{5}) / 2$.

Condition a) of Theorem 3 is satisfied according to Lemma 9. Calculation gives

$$
\begin{array}{cc}
\left(m_{1}, m_{2}, m_{3}\right)=(1,0,-1), & \left(n_{1}, n_{2}, n_{3}\right)=(1,-1,0), \\
\left(p_{1}, p_{2}, p_{3}\right)=(0,1,-1), \quad\left(q_{1}, q_{2}, q_{3}\right)=(1,0,-1) .
\end{array}
$$

One easily makes sure that Eqs. (5.30) with the integers given by (6.38) are independent and have solutions $a=-1, c=d=-b=(1 \pm \sqrt{5}) / 2$. So condition b) of Theorem 3 is also satisfied. Calculations show that conditions (6.19), (6.34) are satisfied as well. Hence Theorem 3 is applicable and gives existence of weak LR for the dual graph formed by the grids.

Since these arguments can be applied to every four of the grids (6.35), (6.36) Proposition 7 is proven.

The existence of weak LR for pentagonal quasicrystals established in Proposition 7 will be used in the next section for the study of three-dimensional quasicrystals having icosahedral symmetry.

\section{Three-Dimensional Quasicrystals}

In this section we show that many of the results obtained in the previous sections can be extended (with appropriate modifications) to three-dimensional quasicrystals. For every tiling of space with parallelotops the dual graph is defined in a way similar to that discussed in Sect. 4. It consists of arrays of two-dimensional surfaces.

The dual graph of a quasicrystalline tiling is topologically equivalent (in the sense of Sect. 4) to $n$ arrays of parallel equidistant planes (three-dimensional grids):

$$
f_{i}(\mathbf{x})=c_{i}+k_{i}
$$

where $i=1, \ldots, n, f_{i} \in\left(\mathbb{R}^{3}\right)^{\prime}, \mathbf{x} \in \mathbb{R}^{3}, c_{i} \in \mathbb{R}, k \in \mathbb{Z}$. We assume that these grids are non-degenerate, i.e. every three of functions $f_{i}$ are linearly independent.

In order to formulate an analogue of Theorem 2 we introduce SI-condition for such grids. Consider four of the grid functions $f_{i}$ from (7.1), say $f_{1}, f_{2}, f_{3}, f_{4}$. We 
say that the grid functions $f_{j}(j=1,2,3,4)$ satisfy an SI-condition, if four integer numbers $k_{j}(j=1,2,3,4)$ exist such that

a) $\left|k_{1}\right|+\left|k_{2}\right|+\left|k_{3}\right|+\left|k_{4}\right|>0$;

b) the equations

$$
f_{j}(\mathbf{x})=k_{j} \quad(j=1,2,3,4)
$$

have a solution.

It proves useful to have the SI-condition (7.2) written in the coordinate representation. Let the functions $f_{j}$ be given by

$$
f_{j}(\mathbf{x})=a_{j} x_{1}+b_{j} x_{2}+c_{j} x_{3},
$$

where $j=1,2,3,4, \mathbf{x}=\left(x_{1}, x_{2}, x_{3}\right) \in \mathbb{R}^{3}$. The SI-condition (7.2) means that

$$
\operatorname{det}\left\|\begin{array}{llll}
a_{1} & b_{1} & c_{1} & k_{1} \\
a_{2} & b_{2} & c_{2} & k_{2} \\
a_{3} & b_{3} & c_{3} & k_{3} \\
a_{4} & b_{4} & c_{4} & k_{4}
\end{array}\right\|=0
$$

After this definition is introduced the generalization of Theorem 2 becomes straightforward.

Theorem 4. If a three-dimensional quasicrystal has strong LR, then for every four of its grids the SI-condition (7.2) is satisfied.

The proof of this theorem is almost identical to the proof of Theorem 2 .

Now we discuss an analogue of Proposition 5. Consider four grids (7.1) and suppose that the condition (7.4) is satisfied for some $k_{j}(j=1,2,3,4)$. The set of all integer vectors $\left(k_{1}, k_{2}, k_{3}, k_{4}\right)$ satisfying (7.4) form a sublattice of the lattice $\mathbb{Z}^{4}$. One should distinguish several possibilities corresponding to the dimension $d$ of this sublattice.

a) If $d=4$, then four vectors $\left(a_{j}, b_{j}, c_{j}\right)(j=1, \ldots, 4)$ belong to a two-dimensional plane (this degenerate case never occurs).

b) If $d=3$, then four grid functions $f_{j}$ are dependent over the field $Q$ :

$$
\sum_{j=1, \ldots, 4} p_{j} f_{j}(\mathbf{x})=0
$$

for all $\mathbf{x} \in \mathbb{R}^{3}$ and some $p_{j} \in \mathbb{Q}$. In this case the corresponding quasicrystal is periodic. $\mathbb{R}^{3}$.

c) If $d=2$, the "second intersection" points form a two-dimensional lattice in

d) If $d=1$, the "second intersection" points form a one-dimensional row.

Consider a three-dimensional quasicrystal produced by $n$ grids. Since every three of the grid functions are linearly independent (see the nondegeneracy condition a) mentioned above), we can choose $f_{1}, f_{2}, f_{3}$ as a basis and express other functions as linear combinations of these three:

$$
f_{j}(\mathbf{x})=a_{j} f_{1}(\mathbf{x})+b_{j} f_{2}(\mathbf{x})+c_{j} f_{3}(\mathbf{x})
$$

$\left(j=4, \ldots, n, \mathbf{x} \in \mathbb{R}^{3}\right)$. Without discussing the whole variety of possibilities we consider two typical examples. 
a) Suppose that for every four of the grid functions the SI-condition is satisfied with $d=1$ (see above). These conditions give $N=C_{n}^{4}=n(n-1)(n-2)(n-3) / 24$ equations of the type (7.4). Suppose further that all these equations are independent. The number $M$ of variables $a_{j}, b_{j}, c_{j}(j=4, \ldots, n)$ is equal to the dimension of the Grassmann manifold $G[n, 3]$, i.e. $M=3(n-3)$. One checks that $M<N$ only when $n>5$. Possible solutions of $N$ equations (7.4) are cubic irrationalities:

$$
a_{j}, b_{j}, c_{j} \in \mathbb{Q}(\sqrt[3]{D}) \text { for all } j \text { and some } D \in \mathbb{Z} \text {. }
$$

b) Suppose that the SI-condition is satisfied for every four grid functions with $d=2$. In this case every SI-condition (7.4) gives two equations for the coefficients $a_{j}$, $b_{j}, c_{j}$ in (7.6), since there are two essentially different ways of choosing the integers $k_{j}$ in (7.2). We assume that all these equations are independent, so the total number of equations is $2 N$. The number of variables is $M$ again. One checks that $M<2 N$ when $n>4$. The solutions of the equations are quadratic irrationalities:

$$
a_{j}, b_{j}, c_{j} \in \mathbb{Q}(\sqrt{D}) \text { for all } j \text { and some } D \in \mathbb{Z} \text {. }
$$

One can think that there exist other possibilities corresponding to the cases intermediate between a) and b).

Possible generalizations of Theorem 3 for the tilings of $\mathbb{R}^{3}$ seem to be rather cumbersome. Instead of making general assertions like Theorem 3 we study a particular example. The quasicrystal having icosahedral symmetry is the most important of three-dimensional quasicrystals, since it has applications to really existing materials (see Sect.1) and is widely discussed in physical literature. By making use of Theorem 3 we show that this quasicrystal satisfies weak LR.

Proposition 8. The icosahedrally symmetric quasicrystalline tiling of $\mathbb{R}^{3}$ satisfies weak LR.

Proof. Consider six 5-fold symmetry axes of the icosahedron and the unit vectors $\mathbf{e}_{i}(i=1, \ldots, 6)$ directed along these axes. The grid functions $f_{i}$ of the icosahedral tiling are given by

$$
f_{i}(\mathbf{x})=\left(\mathbf{e}_{i}, \mathbf{x}\right),
$$

where $\mathbf{x} \in \mathbb{R}^{3}$, " $(\ldots, \ldots)$ " is the standard scalar product in $\mathbb{R}^{3}$. The grids are given by (7.1). Let us choose $r>0$, find the $r$-atlas of the quasicrystal and take this $r$-atlas as local rules.

Consider a tiling of $\mathbb{R}^{3}$ which satisfies these LR, and determine its dual graph. Choose three arrays of surfaces from the dual graph of this tiling, say the first, the second, and the third, and find a continuous transformation $\mathbb{R}^{3} \rightarrow \mathbb{R}^{3}$ which transforms these arrays onto three corresponding grids (as in the proof of Lemma 10). After this transformation is performed the dual graph of the tiling consists of three grids and of three arrays of surfaces which are not necessarily grids. The lifting of this tiling is given by

$$
\begin{aligned}
w(\mathbf{x}) & =\left(w_{1}(\mathbf{x}), \ldots, w_{6}(\mathbf{x})\right), \quad \mathbf{x} \in \mathbb{R}^{3}, \\
w_{j}(\mathbf{x}) & =\left[\left(\mathbf{e}_{j}, \mathbf{x}\right)-c_{j}\right] \quad \text { for } \quad j=1,2,3 .
\end{aligned}
$$


To establish the existence of weak LR we must show that the last three arrays $(j=4,5,6)$ are almost parallel to the corresponding grids.

Let us choose one of the planes of the first grid, say

$$
\left(\mathbf{e}_{1}, \mathbf{x}\right)=c_{1}+k \quad\left(\mathbf{x} \in \mathbb{R}^{3} ; k \in \mathbb{Z}\right),
$$

and consider the intersection lines of this plane and other surfaces of the dual graph. We obtain five arrays of lines on the plane (7.11). Note that threedimensional $r$-rules induce two-dimensional $r^{\prime}$-rules for the so-obtained graph on the plane (7.11), where $r^{\prime}>$ const $r$. These $r^{\prime}$-rules coincide with the $r^{\prime}$-atlas of one of the 5-fold symmetric two-dimensional quasicrystals (6.35), (6.36) with properly chosen constants $c_{j}$. According to Proposition 7 there exist weak LR for this quasicrystal and, moreover, the $r^{\prime}$-atlas gives such rules if $r^{\prime}$ is large enough. This means that the lifting of the two-dimensional tiling, which is given by restricting the last five components of (7.10) on the plane (7.11), is close to the lifting of the quasicrystal:

$$
\left|\left(w_{j}(\mathbf{x})-w_{j}\left(\mathbf{x}^{\prime}\right)\right)-\left(\left(\mathbf{e}_{j}, \mathbf{x}\right)-\left(\mathbf{e}_{j}, \mathbf{x}^{\prime}\right)\right)\right|<\mathrm{const}
$$

for all $x, x^{\prime} \in \mathbb{R}^{3}$ satisfying (7.11) and $j=2, \ldots, 6$. Although the constant in (7.12) does not depend on $k$, condition (7.12) does not imply the existence of threedimensional weak LR. Nevertheless, condition (7.12) means that one can find functions $s_{j}: \mathbb{R} \rightarrow \mathbb{R}(j=2, \ldots, 6)$ such that

$$
\left|w_{j}(\mathbf{x})-\left(\mathbf{e}_{j}, \mathbf{x}\right)-s_{j}(k)\right|<\mathrm{const}
$$

for all $\mathbf{x} \in \mathbb{R}^{3}$ such that $\left(\mathbf{e}_{1}, \mathbf{x}\right)=c_{1}+k, j=2, \ldots, 6$, where the constant in (7.13) does not depend on $k$. Now we choose one of the planes of the second grid, say

$$
\left(\mathbf{e}_{2}, \mathbf{x}\right)=c_{2}+k \quad\left(\mathbf{x} \in \mathbb{R}^{3}, k \in \mathbb{Z}\right),
$$

instead of (7.11) and repeat the above considerations. We obtain that one can find functions $s_{j}^{\prime}: \mathbb{R} \rightarrow \mathbb{R}(j=1,3, \ldots, 6)$ such that

$$
\left|w_{j}(\mathbf{x})-\left(\mathbf{e}_{j}, \mathbf{x}\right)-s_{j}^{\prime}(k)\right|<\mathrm{const},
$$

for all $\mathbf{x} \in \mathbb{R}^{3}$ such that $\left(\mathbf{e}_{2}, \mathbf{x}\right)=c_{2}+k, j=1,3, \ldots, 6$, where the constant does not depend on $k$ again. The conditions (7.13), (7.15) together applied to the components $w_{j}(j=3,4,5,6)$ of the lifting function $w$ give the existence of a constant $C$ such that the inequalities

$$
\begin{aligned}
& \left|w_{j}(\mathbf{x})-\left(\mathbf{e}_{j}, \mathbf{x}\right)-s_{j}\left(\left(\mathbf{e}_{1}, \mathbf{x}\right)\right)\right|<C, \\
& \left|w_{j}(\mathbf{x})-\left(\mathbf{e}_{j}, \mathbf{x}\right)-s_{j}^{\prime}\left(\left(\mathbf{e}_{2}, \mathbf{x}\right)\right)\right|<C,
\end{aligned}
$$

are satisfied simultaneously for all $j$. Consequently

$$
\left|s\left(\left(\mathbf{e}_{1}, \mathbf{x}\right)\right)-s^{\prime}\left(\left(\mathbf{e}_{2}, \mathbf{x}\right)\right)\right|<2 C
$$

for all $\mathbf{x} \in \mathbb{R}^{3}$ and $j=3,4,5,6$. This implies that both $s_{j}(\mathbf{x})$ and $s_{j}^{\prime}(\mathbf{x})$ is uniformly bounded on $\mathbb{R}^{3}$ for all $j$. We have proven that the functions $w_{j}(\mathbf{x})-\left(\mathbf{e}_{j} \cdot \mathbf{x}\right)$ are bounded on $\mathbb{R}^{3}$ for $j=3,4,5,6$. The proof for $j=1,2$ is quite analogous.

The existence of the weak LR for icosahedral quasicrystals is established. 


\section{Concluding Remarks}

The results of this work give strong evidence that strong LR for a planar quasicrystal exist only if it is generated by some quadratic irrationalities. To prove this rigorously one has to exclude the "exceptional" case 2 of Propositions 5, 6. Moreover, one can believe that quasicrystals in an arbitrary dimension $d$ can have strong LR only when they are generated by irrational numbers which are roots of polynomials of power not more than $d$.

It is interesting to note that all the quasicrystals found in experiments are based on quadratic irrationalities. There are four types of symmetry among the quasicrystalline materials known so far:

a) icosahedral symmetry based on the field $\mathbb{Q}(\sqrt{5})[1]$;

b) pentagonal symmetry based on the same field $\mathbb{Q}(\sqrt{5})[17]$;

c) dodecagonal symmetry based on the field $\mathbb{Q}(\sqrt{3})[18]$;

d) octagonal symmetry based on the field $\mathbb{Q}(\sqrt{2})[19]$.

One can speculate that the reason for the absence of quasicrystals with other irrationalities is that only quadratic irrationalities provide local rules.

Several problems on quasicrystalline tilings not yet studied should be mentioned. The first of them is the relationship between strong LR and weak LR. It is more or less clear from Theorems 1,2, 3 which planar quasicrystals cannot have strong LR and which have weak LR. But which of them have strong LR? The example by de Bruijn and the counterexample by Beenker show that the problem is rather difficult. Nevertheless some work should be done to find a generalization of de Bruijn's method for other quadratic irrationalities.

Another interesting problem concerns the properties of quasicrystals which have weak LR, but do not have strong LR. Weak LR leave some arbitrariness in the tiling. How large is this freedom? One can expect that there are of order $\exp (\alpha S), \alpha>0$, different tilings of a large region of the area $S$ and the ground state of such quasicrystals has nonzero entropy per a unit area. Has this any physical consequences?

It is very interesting also to reach some more understanding of properties of three-dimensional quasicrystals. The analogues of Theorem 3 and Propositions 5, 6 should be found. Which three-dimensional quasicrystals have strong LR?

Acknowledgements. I am grateful to A. Pavlovitch for an interesting talk which drew my attention to the tilings of the plane. Many interesting discussions with A.Yu.Kitaev, A.Shen, and P. A. Kalugin were extremely useful for me. I am indebted to V.L.Pokrovsky, Ya. G. Sinai, and S. E. Burkov for their interest in this work and constant encouragement. I would like to thank Ya. G. Sinai for helpful advice concerning the manuscript.

\section{References}

1. Shechtman, D., Blech, I.A., Gratias, D., Cahn, J.W.: Phys. Rev. Lett. 53, No. 20, 1951-1953 (1984)

2. Kalugin, P., Kitaev, A., Levitov, L.: Sov. Phys. JETP Lett. 41, 145 (1985); J. Phys. Lett. 46, L601, (1985)

3. Elser, V.: Phys. Rev. B 32, 4892 (1985) 
4. Duneau, M., Katz, A.: Phys. Rev. Lett. 54, 2688-2691 (1985)

5. Mackay, A.: Physica 114 A, 609-613 (1982)

6. Levine, D., Steinhardt, P.J.: Phys. Rev. Lett. 53, 2477 (1984)

7. Kramer, P., Neri, R.: Acty. Cryst. A 40, 580-587 (1984)

8. Penrose, R.: Bull. Inst. Math. Appl. 10, 266-271 (1974)

9. Galer, F., Rhyner, J.: J. Phys. A: Math. Gen. 19, 267-277 (1986)

10. Henley, C.L., Elser, V.: Phil. Mag. Lett. 53 No. 3, 59-66 (1986)

11. de Bruijn, N.G.: Nederl. Akad. Wetensch. Proc. Ser. A, 84, 38-66 (1981)

12. Kleman, M., Pavlovitch, A.: J. Phys. C3, Workshop on Aperiodic Crystals, 229 (1986)

13. Beenker, F.: Eindhoven University of Technology, Department of Mathematics and Computing Science. TH-Report 82-WSK-04 Sept. 1982

14. Levitov, L.S.: Zh. Eksp. Teor. Fiz. 93, 1832 (1987)

15. Landau, L.D.: In: Collected papers translated. Haar, D. Ter, pp. 540-545; New York: Gordon and Breach 1965; see also Burkov, S.E.: J. Phys. 46, 317 (1985)

16. Harary, F.: Graph Theory. Reading, MA: Addison-Wesley 1969

17. Bendersky, L.: Phys. Rev. Lett. 55, 1461 (1985)

18. Ishimasa, T., Nissen, H-U., Fukano, Y.: Phys. Rev. Lett. 55, 511 (1985)

19. Wang, N., Chen, H., Kuo, K.H.: Phys. Rev. Lett. 59, 1010 (1987)

20. Cassels, J.W.S.: An Introduction to Diofantine Approximation (Chap. 3, Sect. 5), Cambridge University Press 1957

Communicated by Ya. G. Sinai

Received April 15, 1988

Note added in proof. In a recent paper Burkov shows that weak local rules are absent for a twodimensional quasicrystal having 8-fold symmetry (see the next paper in this issue). In this Note I would like to comment on the relation of Burkov's result and Theorem 3. Grid functions for the quasicrystal studied by Burkov are given by

$$
\begin{gathered}
f_{1}(x)=x_{1}, \quad f_{2}(x)=\lambda\left(x_{1}+x_{2}\right), \quad f_{3}(x)=x_{2}, \quad f_{4}(x)=\lambda\left(x_{2}-x_{1}\right), \\
\lambda=1 / \sqrt{2}, \quad x=\left(x_{1}, x_{2}\right) \in \mathbb{R}^{2} .
\end{gathered}
$$

One can check that SI-conditions are satisfied for all triplets of functions $f_{i}(i=1,2,3,4)$. The corresponding triplets of integer numbers in (5.30) are

$$
\begin{gathered}
\left(m_{1}, m_{2}, m_{3}\right)=(1,0,-1), \quad\left(n_{1}, n_{2}, n_{3}\right)=(0,1,1), \\
\left(p_{1}, p_{2}, p_{3}\right)=(1,1,0), \quad\left(q_{1}, q_{2}, q_{3}\right)=(1,0,-1) .
\end{gathered}
$$

However Eqs. (5.30) with $m_{j}, n_{j}, p_{j}, q_{j}$ from (N.2) are not independent, i.e. they correspond to Case 2 of Proposition 5. In accordance with this proposition the solutions of Eqs. (5.30) form a oneparametric set (N.1), where $\lambda$ is not $1 / \sqrt{2}$ but an arbitrary real number. This set of functions is used in Burkov's work. Absence of local rules does not contradict Theorem 3 since Eqs. (5.30) are not independent. Moreover, it confirms our expectation (see the note after Proposition 6).

What about other 8-fold symmetric quasicrystals? Let us show that Burkov's result is not generic, i.e. there exist 8 -fold symmetric quasicrystals having local rules. Consider 8 grid functions $f_{i} \in\left(\mathbb{R}^{2}\right),(i=1, \ldots, 8)$ such that $f_{1}, f_{2}, f_{3}, f_{4}$ are given by (N.1) while other functions are defined as

$$
f_{5}=\mu f_{1}+f_{2}, \quad f_{6}=\mu f_{2}+f_{3}, \quad f_{7}=\mu f_{3}+f_{4}, \quad f_{8}=\mu f_{4}-f_{1},
$$


where $\mu=\mu^{\prime}+\mu^{\prime \prime} \sqrt{2} \in \mathbb{Q}(\sqrt{2}),\left(\mu^{\prime}, \mu^{\prime \prime} \in \mathbb{Z}\right)$. Note that the grid pattern generated by the functions $f_{1}, \ldots, f_{8}$ has octagonal symmetry. Taking $f_{1}=x_{1}, f_{2}=x_{2}$ as a basis of $\left(\mathbb{R}^{2}\right)^{\prime}$ one can express all $f_{i}$ $(i=1, \ldots, 8)$ as

$$
f_{t}(x)=a f_{1}(x)+b f_{2}(x), \quad a, b \in \mathbb{Q}(\sqrt{2}),
$$

Choose four functions $f_{1}, f_{3}, f_{2}, f_{5}$ and write SI-conditions for them. SI-conditions are satisfied by the triplets of integers given as

1) triplet $\left(f_{1}, f_{3}, f_{2}\right)-\left(m_{1}, m_{2}, m_{3}\right)=(1,-1,0)$;

2) triplet $\left(f_{1}, f_{3}, f_{5}\right)-\left(n_{1}, n_{2}, n_{3}\right)=\left(1,-1-\mu^{\prime \prime}, \mu^{\prime}\right)$;

3) triplet $\left(f_{1}, f_{2}, f_{5}\right)-\left(p_{1}, p_{2}, p_{3}\right)=(0,1,1)$;

4) triplet $\left(f_{3}, f_{2}, f_{5}\right)-\left(q_{1}, q_{2}, q_{3}\right)=\left(\mu^{\prime}, 2 \mu^{\prime \prime}, 2 \mu^{\prime \prime}\left(1+\mu^{\prime \prime}\right)-\mu^{\prime 2}\right)$.

One can check that Eqs. (5.30) with $m_{j}, n_{j}, p_{j}, q_{j}(j=1,2,3)$ taken from (N.5) are independent whenever $\mu^{\prime} \neq 0, \mu^{\prime \prime} \neq 0$. This suggests to apply Theorem 3 . Simple calculations show that conditions (6.19), (6.34) are satisfied for the triplets (N.5) when $\mu^{\prime} \neq 0, \mu^{\prime \prime} \neq 0$. Thus Theorem 3 is applicable and we find that weak local rules exist for the tiling based on the grids $f_{1}, f_{3}, f_{2}, f_{5}$.

As for functions $f_{4}, f_{6}, f_{7}, f_{8}$, they can be expressed as rational linear combinations

$$
f_{k}=a_{k} f_{1}+b_{k} f_{3}+c_{k} f_{2}+d_{k} f_{5},
$$

where $a_{k}, b_{k}, c_{k}, d_{k} \in \mathbb{Q}, k=4,6,7,8$. So the tiling corresponding to $f_{1}, \ldots, f_{8}$ can be considered as a "decoration" of that corresponding to $f_{1}, f_{3}, f_{2}, f_{5}$. By this reason weak LR exist for eight grids $f_{1}, \ldots, f_{8}$.

We see that the example by Burkov is not a typical one. Weak local rules are absent for 8-fold symmetric quasicrystals only when the grids are very specially chosen. For a generic set of grids having 8 -fold symmetry weak LR do exist.

In conclusion I would like to thank S. E. Burkov for giving me an opportunity to become aware of his work before publication. 